\title{
ENTROPY STABLE ESSENTIALLY NONOSCILLATORY METHODS BASED ON RBF RECONSTRUCTION
}

\author{
JAn S. Hesthaven AND FABIAN MÖNKEBERG*
}

\begin{abstract}
To solve hyperbolic conservation laws we propose to use high-order essentially nonoscillatory methods based on radial basis functions. We introduce an entropy stable arbitrary high-order finite difference method (RBF-TeCNOp) and an entropy stable second order finite volume method (RBFEFV2) for one-dimensional problems. Thus, we show that methods based on radial basis functions are as powerful as methods based on polynomial reconstruction. The main contribution is the construction of an algorithm and a smoothness indicator that ensures an interpolation function which fulfills the sign-property on general one dimensional grids.
\end{abstract}

Mathematics Subject Classification. 35L65, 65M12, 65M06, 65M08, 65D05.

Received August 13, 2018. Accepted February 11, 2019.

\section{INTRODUCTION}

Conservation laws arise in different fields of physics to describe systems with certain conserved properties, e.g. mass, momentum, and energy. A change in these properties within a domain can be described by the flux through its boundaries. The one-dimensional conservation law on differential form is given as

$$
\begin{aligned}
u_{t}+f(u)_{x} & =0, \quad(x, t) \in \mathbb{R} \times \mathbb{R}_{+}, \\
u(0) & =u_{0},
\end{aligned}
$$

with the conserved variables $u: \mathbb{R} \times \mathbb{R}_{+} \rightarrow \mathbb{R}^{N}$ and the flux $f: \mathbb{R}^{N} \rightarrow \mathbb{R}^{N}$. A well known and challenging property is the formation of discontinuities out of smooth initial data [25]. Thus, solutions need to be defined in the weak (distributional) sense. Since the weak solutions are not unique they need to be restricted by additional conditions. Let $\eta$ be a convex scalar function (entropy function) such that there exists the entropy flux $q$ with $\nabla_{u} q=\nabla_{u} \eta \nabla_{u} f$. The function $u: \mathbb{R} \times \mathbb{R}_{+} \rightarrow \mathbb{R}^{N}$ is called an entropy solution of (1.1) for the entropy pair $(\eta, q)$ if the inequality

$$
\eta(u)_{t}+q(u)_{x} \leqslant 0
$$

is satisfied in a weak sense. In the case of scalar conservation laws, existence and uniqueness of the weak entropy solution in $\mathbb{R}^{d}$ was shown by Kružkov [21]. Furthermore, we can use entropy variables $v:=\left(\nabla_{u} \eta\right)^{T}$ to symmetrize

Keywords and phrases. Radial basis functions, entropy stability, sign-property, finite differences, finite volume methods, high-order accuracy, ENO reconstruction.

SB-MATHICSE-MCSS, École Polytechnique Fédérale de Lausanne (EPFL), 1015 Lausanne, Switzerland.

*Corresponding author: fabian.monkeberg@epfl.ch 
(1.1) in the sense that $\nabla_{v} u(v)$ is symmetric positive definite and $\nabla_{v} f(u(v))$ is symmetric. This can be seen by introducing the entropy potential $\psi(v)=v^{T} \cdot f(u(v))-q(u(v))$ to recover $\nabla_{v} f(u(v))=\nabla_{v v} \psi$ [28]. Note that entropy solutions satisfy

$$
\eta(u)_{t}+q(u)_{x}=0
$$

in smooth regions, but satisfy (1.2) at discontinuities as entropy must dissipate.

\subsection{Finite difference and finite volume methods}

One goal of numerical methods is to express the approximative behaviour of the physical correct solution.

Let us assume a one-dimensional grid $\left\{x_{i}\right\}_{i \in \mathbb{Z}} \subset \mathbb{R}$, partitioned into cells $C_{i}=\left[x_{i-1 / 2}, x_{i+1 / 2}\right]$ of size $\Delta x$. The finite difference approach is based on approximating spatial derivatives at $x_{i}$ as

$$
\frac{\partial f}{\partial x}\left(u_{i}\right)=\frac{F_{i+1 / 2}-F_{i-1 / 2}}{\Delta x}+\mathcal{O}\left(\Delta x^{p}\right)
$$

with $p>0$. This results in the semi-discrete scheme

$$
\frac{\mathrm{d} u_{i}}{\mathrm{~d} t}+\frac{1}{\Delta x}\left(F_{i+1 / 2}-F_{i-1 / 2}\right)=0
$$

where the numerical flux terms $F_{i+1 / 2}$ depend on point values $\left\{u_{i-k}, \ldots, u_{i+p-k}\right\}$ with $0 \leqslant k \leqslant p-1$.

On the other hand, finite volume methods work with mean values $\bar{u}_{i}$ for the cells $C_{i}$. By integrating (1.1) over the cells and dividing it by its size $\left|C_{i}\right|$ we recover (1.5) with the difference that

$$
f\left(u\left(x_{i+1 / 2}\right)\right)=F_{i+1 / 2}+\mathcal{O}\left(\Delta x^{p}\right) .
$$

There exist multiple high-order accurate methods to solve conservation laws, for example the MUSCL scheme introduced in [39], the ENO scheme [18] or the WENO scheme [37]. In all cases, we can apply an arbitrary time discretization technique to recover a fully discrete scheme, e.g. an SSPRK method.

In [11], Fjordholm et al. proposed an entropy stable TeCNO scheme based on polynomial reconstruction. We follow the spirit of this work and introduce a scheme based on radial basis functions (RBF). Note that for taking advantage of the RBFs on general grids in higher dimensions, we would need a MUSCL type scheme.

In Section 2 we introduce the framework of entropy stable schemes based on an entropy conservative flux and a diffusion operator [11,38]. We describe the basics of RBFs in Section 3. Furthermore, we give an explicit representation of the interpolation function for infinitely smooth RBFs in one dimension. Sections 4 and 5 contain the main contribution. In Section 4 we introduce a smoothness indicator for RBFs which is based on the generalized divided difference method and in Section 5 we prove that it fulfills a certain stability property (the sign-property) for general grids in one dimension. Section 6 combines these results to construct an arbitrarily high-order RBF based entropy stable finite difference method and a second order entropy stable finite volume one that is generalizable to general grids in higher dimensions. In the last section we demonstrate the robustness of the numerical scheme with a variety of one dimensional examples.

\section{ENTROPY STABLE METHOdS}

The goal is to construct methods that fulfill a discrete version of (1.2), referred to as entropy stable [19,23]. As a first step, we introduce entropy conservative methods that fulfill (1.3) at the discrete level. Next, we add a dissipation term to control oscillations at discontinuities to recover an entropy stable method. 


\subsection{Entropy conservative methods}

A finite difference method is entropy conservative if it satisfies

$$
\frac{\mathrm{d}}{\mathrm{d} t} \eta\left(u_{i}\right)=-\frac{1}{\left|C_{i}\right|}\left[Q_{i+1 / 2}-Q_{i-1 / 2}\right]
$$

for a consistent numerical entropy flux $Q_{i+1 / 2}$. To construct entropy conservative methods we use Tadmor's entropy conservation condition [38]

$$
\llbracket v \rrbracket_{i+1 / 2}^{T} F_{i+1 / 2}=\llbracket \psi \rrbracket_{i+1 / 2} .
$$

This condition describes a system of equations, but its solvability is not clear. For scalar conservation laws there exists a unique solution as can be summarized in the following theorem.

Theorem 2.1 (Entropy conservative schemes for scalar equations [38]). For a given entropy pair $(\eta, q)$ the numerical flux

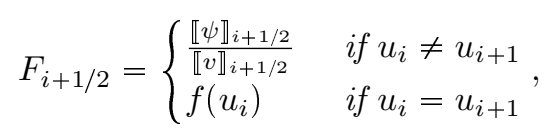

defines an entropy conservative method for scalar equations with the entropy variable $v$ and the conserved variable $u$. Furthermore, it is second-order accurate in smooth regions of $u$.

Given a numerical second order two-point flux the work of Lefloch et al. [24] combines these linearly to construct a $2 p$ th order accurate flux on a uniform grid.

Theorem 2.2 (High-order entropy conservative fluxes [24]). Let $p \in \mathbb{N}$ and assume that $\alpha_{1, p}, \ldots, \alpha_{p, p}$ solve the $p$ linear equations

$$
\sum_{i=1}^{p} i \alpha_{i, p}=1, \quad \sum_{i=1}^{p} i^{2 s-1} \alpha_{i, p}=0, \quad \text { for } s=2, \ldots, p .
$$

Then the flux

$$
\tilde{F}^{2 p}\left(u_{i-p+1}, \ldots, u_{i+p}\right)=\sum_{j=1}^{p} \alpha_{j, p} \sum_{l=1}^{j} \tilde{F}^{2}\left(u_{i-j+l}, u_{i+l}\right),
$$

is consistent, $2 p$ th order accurate and entropy conservative provided the second order two-point conservative flux $\tilde{F}^{2}$ fulfills (2.2).

The fourth order entropy conservative flux with coefficients $\alpha_{2}=\left(\frac{4}{3},-\frac{1}{6}\right)$ and the sixth order scheme with $\alpha_{3}=\left(\frac{3}{2},-\frac{3}{10}, \frac{1}{30}\right)$ present two explicit examples.

\subsubsection{Entropy conservative methods for shallow water equations}

The shallow water equations describe a flow under the assumptions that the horizontal length scales are much larger than the vertical ones. In one space dimension the system of equations depends on the mass flow $m$ and the fluid height $h$

$$
\left(\begin{array}{c}
h \\
m
\end{array}\right)_{t}+\left(\begin{array}{c}
m \\
\frac{1}{2} g h^{2}+m^{2} / h
\end{array}\right)_{x}=0
$$

with the gravitational constant $g$ [25]. To apply Theorem 2.2 we need to construct a second order entropy conservative scheme by solving (2.2). One choice of an entropy pair for the one-dimensional shallow water equation is

$$
\eta=\frac{1}{2}\left(\frac{m^{2}}{h}+g h^{2}\right), \quad q=\frac{m^{3}}{h^{2}}+g m h,
$$


which results in the entropy variables and the potential

$$
v=\left(g h-\frac{m^{2}}{\frac{m}{h}}\right), \quad \psi=\frac{1}{2} g m h .
$$

An alternative second order entropy conservative flux is

$$
\tilde{F}_{i+1 / 2}=\left(\begin{array}{c}
\bar{h}_{i+1 / 2} \bar{u}_{i+1 / 2} \\
\bar{h}_{i+1 / 2}\left(\bar{u}_{i+1 / 2}\right)^{2}+\frac{1}{2} g \bar{h}^{2}{ }_{i+1 / 2}
\end{array}\right)
$$

with $u=m / h$ and $\bar{f}_{i+1 / 2}=\frac{1}{2}\left(f_{i}+f_{i+1}\right)[10]$.

\subsubsection{Entropy conservative methods for Euler equations}

The Euler equations can be recovered from the Navier-Stokes equations by neglecting the viscosity. They consist of the continuity equation, momentum equation and the conservation law for the total energy. In one dimension they are

$$
\left(\begin{array}{c}
\rho \\
m \\
E
\end{array}\right)_{t}+\left(\begin{array}{c}
m \\
\frac{m^{2}}{\rho}+p \\
\frac{m}{\rho}(E+p)
\end{array}\right)_{x}=0,
$$

with $p=\mathcal{R} \rho T=(\gamma-1)\left(E-\frac{1}{2} \frac{m^{2}}{\rho}\right)$ for an ideal gas with the ratio of specific heat $\gamma[20]$. For the Euler equations the thermodynamical entropy $s=\log (p)-\gamma \log (\rho)$ is different from the entropy function and the entropy flux. One possible pair is proposed in [11]

$$
\eta=\frac{-\rho s}{\gamma-1}, \quad q=\frac{-m s}{\gamma-1} .
$$

Chandrashekar [5] proposed the kinetic energy preserving and entropy conservative (KEPEC) flux, based on the entropy variables and the potential

$$
v=\left(\begin{array}{c}
\frac{\gamma-s}{\gamma-1}-\frac{\rho u^{2}}{2 p} \\
\rho u / p \\
-\rho / p
\end{array}\right), \quad \psi=\rho u .
$$

The KEPEC flux makes use of the logarithmic averages $\hat{\rho}$ and $\hat{\beta}$ with $\beta=\frac{\rho}{2 p}$ and can be written as

$$
f^{\rho}=\hat{\rho} \bar{u}, \quad f^{m}=\frac{\bar{\rho}}{2 \bar{\beta}}+\bar{u} f^{\rho}, \quad f^{e}=\left(\frac{1}{2(\gamma-1) \hat{\beta}}-\frac{1}{2} \overline{u^{2}}\right) f^{\rho}+\bar{u} f^{m}
$$

where $\bar{v}=\frac{v_{i+1}+v_{i}}{2}$ and with the logarithmic average defined as $\hat{v}=\frac{v_{i}-v_{i+1}}{\log \left(v_{i}\right)-\log \left(v_{i+1}\right)}$.

\subsection{Entropy stable methods}

Entropy conservative methods yield good results in smooth regions, but it is well-known that spurious oscillations appear close to discontinuities. Introducing artificial dissipation, depending on the size of the jump in the interface, controls these oscillations. Based on an entropy conservative scheme $\tilde{F}_{j+1 / 2}$ of second order and a symmetric positive definite matrix $D_{i+1 / 2}$, Tadmor constructed the entropy stable numerical flux function [38]

$$
F_{i+1 / 2}=\tilde{F}_{j+1 / 2}-\frac{1}{2} D_{i+1 / 2} \llbracket v \rrbracket_{i+1 / 2} .
$$

Combining high-order conservative fluxes with dissipation terms introduces the constraint that $D_{i+1 / 2} \llbracket v \rrbracket_{i+1 / 2}=\mathcal{O}\left(\Delta x^{p}\right)$ to maintain accuracy for smooth solutions. 
For each cell $C_{i}$ we define a stencil of cells $S_{i}$ on which we construct an interpolation function $s_{i}(x)$ of order $p$ and replace the jump $\llbracket v \rrbracket_{i+1 / 2}$ by the jump in the reconstruction $\langle v\rangle_{i+1 / 2}=s_{i+1}\left(x_{i+1 / 2}\right)-s_{i}\left(x_{i+1 / 2}\right)$. Thus, the method has the form

$$
F_{i+1 / 2}=\tilde{F}_{i+1 / 2}^{2 p}-\frac{1}{2} D_{i+1 / 2}\left\langle\langle v\rangle_{i+1 / 2},\right.
$$

with the additional condition

$$
D_{i+1 / 2}=R_{i+1 / 2} \Lambda_{i+1 / 2} R_{i+1 / 2}^{T},
$$

where $R_{i+1 / 2} \in \mathbb{R}^{N \times N}$ is invertible and $\Lambda_{i+1 / 2} \geqslant 0$ is diagonal. Fjordholm et al. recovered the following stability results [11].

Lemma 2.3 (Entropy stability with high-order diffusion [11]). For each $i \in \mathbb{Z}$, let (2.16) be fulfilled. Let $s_{i}$ be a reconstruction of the entropy variables in cell $C_{i}$, such that for each $i$, there exists a diagonal matrix $B_{i+1 / 2} \geqslant 0$ such that

$$
\langle v\rangle_{i+1 / 2}=R_{i+1 / 2}^{-T} B_{i+1 / 2} R_{i+1 / 2}^{T} \llbracket v \rrbracket_{i+1 / 2} .
$$

Then the scheme with the flux (2.15) is entropy stable.

By introducing the scaled entropy variables

$$
w_{i}^{ \pm}=R_{i \pm 1 / 2}^{T} v_{i}, \quad \tilde{w}_{i}^{ \pm} R_{i \pm 1 / 2}^{T} v_{i}^{ \pm},
$$

with the reconstructed entropy variables $v_{i}^{ \pm}=s_{i}\left(x_{i \pm 1 / 2}\right),(2.17)$ becomes

$$
\langle\tilde{w}\rangle_{i+1 / 2}=B_{i+1 / 2} \llbracket w \rrbracket_{i+1 / 2} .
$$

Since $B_{i+1 / 2}$ is diagonal and semi-positive definite, this can be reformulated componentwise as

$$
\operatorname{sgn}\left\langle\left\langle\tilde{w}^{l}\right\rangle_{i+1 / 2}=\operatorname{sgn} \llbracket w^{l} \rrbracket_{i+1 / 2},\right.
$$

for each component $l$. This structural property of the reconstruction is called the sign-property.

\subsection{Entropy stable finite volume methods}

The setting of the one dimensional finite volume method differs only slightly from the finite difference scheme, i.e. we consider cell-average values $\bar{u}_{i}$ rather than point values and the definition of higher order methods changes to

$$
F_{i+1 / 2}=f\left(u\left(x_{i+1 / 2}\right)\right)+\mathcal{O}\left(\Delta x^{p}\right) .
$$

Nevertheless, given a 2-point second order finite difference flux $F$, it is also a second order accurate finite volume flux, which follows from the midpoint rule.

Since the definition of entropy conservative schemes does not change for finite volume methods, we can conclude that a second order finite difference flux that fulfills (2.2) is also a second order entropy conservative finite volume method. This can be summarized as follows.

Theorem 2.4. Every second order finite difference scheme that fulfills Tadmor's entropy conservation condition (2.2) in one space dimension is also a second order entropy conservative finite volume method.

The construction of entropy stable schemes from entropy conservative schemes proceeds as for the finite difference case, the only difference being that the interpolation is based on cell-averages instead of point values.

Thus, Lemma 2.3 holds also for finite volume methods and we recover an entropy stable finite volume method of the form

$$
F_{i+1 / 2}=F_{i+1 / 2}^{2}-D_{i+1 / 2}\left\langle\langle v\rangle_{i+1 / 2} .\right.
$$

Note that the extension to higher order as in Theorem 2.2 does not work in the finite volume case and we are not aware of any method doing it. 


\section{RADIAL BASIS FUNCTIONS}

Radial basis functions (RBF) are successfully used for scattered data interpolation. Due to their mesh-free property, they are more flexible in terms of the geometric structure of the data points. Furthermore, their application to high-dimensional problems is immediate. Following the seminal work by Duchon [8] and Micchelli [27], RBFs are successfully used in different domains.

\subsection{Basic interpolation}

The goal is the interpolation of a data vector $\left.f\right|_{X}=\left(f\left(x_{1}\right), \ldots, f\left(x_{n}\right)\right)^{T} \in \mathbb{R}^{n}$, defined at a scattered set of data points $X=\left(x_{1}, \ldots, x_{n}\right)^{T}$ with $x_{i} \in \mathbb{R}^{d}$ for some function $f: \mathbb{R}^{d} \rightarrow \mathbb{R}$. The basic idea is to use one univariate continuous function $\phi$, the radial basis function, composed with the Euclidean norm centered at the data points as the interpolation basis

$$
\mathcal{B}=\left\{\phi\left(\varepsilon\left\|x-x_{1}\right\|\right), \ldots, \phi\left(\varepsilon\left\|x-x_{n}\right\|\right)\right\},
$$

parametrized by the shape parameter $\varepsilon$. To simplify the notation we use

$$
\phi\left(x-x_{i}\right):=\phi\left(\varepsilon\left\|x-x_{i}\right\|\right), \quad \phi: \mathbb{R}^{d} \rightarrow \mathbb{R} .
$$

The standard radial basis function approximation is written as

$$
s(x)=\sum_{i=1}^{n} a_{i} \phi\left(x-x_{i}\right)+p(x)
$$

with a polynomial $p \in \Pi_{m-1}\left(\mathbb{R}^{d}\right), m \in \mathbb{N}$, the interpolation condition

$$
s\left(x_{i}\right)=f\left(x_{i}\right),
$$

and the additional constraints

$$
\sum_{i=1}^{n} a_{i} q\left(x_{i}\right)=0, \quad \text { for all } q \in \Pi_{m-1}\left(\mathbb{R}^{d}\right),
$$

with the coefficients $a_{i} \in \mathbb{R}$ for all $i=1, \ldots, n$. Conditions (3.4) and (3.5) can be expressed in the system of equations

$$
\left(\begin{array}{cc}
A & P \\
P^{T} & 0
\end{array}\right)\left(\begin{array}{l}
a \\
b
\end{array}\right)=\left(\begin{array}{c}
\left.f\right|_{X} \\
0
\end{array}\right)
$$

The choice of the radial basis function $\phi$ is restricted to insure the solvability of (3.6).

Definition 3.1 (Conditionally positive function). A function $\phi: \mathbb{R}^{d} \rightarrow \mathbb{R}$ is called conditionally positive (semi-) definite of order $m$ if, for any pairwise distinct points $x_{1}, \ldots, x_{n} \in \mathbb{R}^{d}$ and $c=\left(c_{1}, \ldots, c_{n}\right)^{T} \in \mathbb{R}^{n} \backslash\{0\}$ such that

$$
\sum_{i=1}^{n} c_{i} p\left(x_{i}\right)=0
$$

for all $p \in \Pi_{m-1}\left(\mathbb{R}^{d}\right)$, the quadratic form

$$
\sum_{j, k=1}^{n} c_{j} c_{k} \phi\left(x_{j}-x_{k}\right)
$$

is positive (non-negative). 
TABle 1. Commonly used RBFs with $\mathbb{N} \nexists \nu>0, k \in \mathbb{N}$ and $\varepsilon>0$.

\begin{tabular}{lll}
\hline \hline RBF & $\phi(r)$ & Order \\
\hline Infinitely smooth RBFs & & \\
Multiquadratics & $\left(1+(\varepsilon r)^{2}\right)^{\nu}$ & $\lceil\nu\rceil$ \\
Inverse multiquadratics & $\left(1+(\varepsilon r)^{2}\right)^{-\nu}$ & 0 \\
Gaussians & $\exp \left(-(\varepsilon r)^{2}\right)$ & 0 \\
\hline Piecewise smooth RBFs & & \\
Polyharmonic Splines & $r^{2 k-d}$ & $k$ \\
& $r^{2 k-d} \log (r)$ & $k$ \\
\hline
\end{tabular}

Wendland shows in [40] that for a conditionally positive definite RBF $\phi$ of order $m$ (3.6) has a unique solution if $x_{1}, \ldots, x_{n}$ are $\Pi_{m-1}\left(\mathbb{R}^{d}\right)$-unisolvent.

Definition 3.2 (Positive definite functions). A function $\phi: \mathbb{R}^{d} \rightarrow \mathbb{R}$ is called positive definite if the quadratic form

$$
\sum_{j, k=1}^{n} c_{j} c_{k} \phi\left(x_{j}-x_{k}\right)
$$

is positive for any $n$ pairwise different points $x_{1}, \ldots, x_{n} \in \mathbb{R}^{d}$ and $c=\left(c_{1}, \ldots, c_{n}\right)^{T} \in \mathbb{R}^{n} \backslash\{0\}$.

Note that for a positive definite function $\phi$, the matrix $A$ is positive definite and there exists an unique solution $a$ to $(3.6)$.

Some examples of positive definite functions are the inverse multiquadratics and the Gaussians (Tab. 1). Other RBFs fulfill a slightly weaker condition and are conditionally positive definite of order $k$, e.g. multiquadratics and polyharmonic splines.

\subsection{Interpolation of cell-averages}

For the finite volume method we do not consider the pointwise interpolation, but cell-averages. Let us assume a given grid of cells $C_{1}, \ldots, C_{n}$ with its average values $\bar{u}_{1}, \ldots, \bar{u}_{n}$ for $n \in \mathbb{N}$. Following [1,2] we consider

$$
s(x)=\sum_{i=1}^{n} a_{i} \lambda_{C_{i}}^{\xi} \phi(x-\xi)+p(x), \quad p \in \Pi_{m-1}\left(\mathbb{R}^{d}\right),
$$

with the average operator of $f$ over the cell $C, \lambda_{C}^{\xi} f$, such that

$$
\begin{aligned}
\lambda_{C_{j}} s & =\bar{u}_{j}, & \text { for all } j=1, \ldots, n, \\
\sum_{i=1}^{n} a_{i} \lambda_{C_{i}}(q) & =0, & \text { for all } q \in \Pi_{m-1}\left(\mathbb{R}^{d}\right) .
\end{aligned}
$$

To show solvability of system (3.11) it suffices to assume that $\phi$ is conditionally positive definite in a pointwise sense.

Aboiyar et al. prove in [1] that (3.11) has a unique solution if the set $\left\{\lambda_{C_{i}}\right\}_{i=1}^{n}$ is $\Pi_{m-1}\left(\mathbb{R}^{d}\right)$-unisolvent.

Theorem 3.3 (Well-posedness of RBF interpolation in the mean value sense). Let $\phi$ be a conditionally positive definite radial basis function and let the set $\left\{\lambda_{C_{i}}\right\}_{i=1}^{n}$ be $\Pi_{m-1}\left(\mathbb{R}^{d}\right)$-unisolvent with $n \in \mathbb{N}$. Then, the problem (3.11) has a unique solution. 
The proof closely follows the one for the pointwise evaluation in [40] plus an estimate for the positive definiteness, based on a pointwise result in [31].

Despite the simple extension of RBF-interpolation in multiple dimensions, there is a major drawback, often referred to as the Uncertainty Principle [34]. It refers to the trade-off between the well-known properties that flat infinitely smooth $\mathrm{RBFs}(\varepsilon \rightarrow 0)$ have an increasing approximation power but a decreasing numerical stability due to ill-conditioning of the interpolation matrix $[7,22,36]$. To overcome the ill-conditioning there are multiple propositions for choosing an "optimal" shape parameter $[9,33]$. Note that a continuous scaling $\varepsilon=\alpha n^{-1 / d}$ causes error stagnation [4]. However, there are multiple approaches which overcome this problem: the RBF-CP [16], the RBF-QR [15], and the RBF-GA [14]. We decided to use the more flexible vector-valued rational approximation method (RBF-RA), based on the RBF-CP algorithm [42].

\subsection{Explicit formula of the RBF interpolation}

Let us consider the pointwise RBF interpolation problem (3.6) with the interpolation function (3.3). Furthermore, let $x_{1}, \ldots, x_{n}$ be the grid points such that $x_{i}<x_{i+1}$ and $n \in \mathbb{N}$ and $y_{1}, \ldots, y_{n} \in \mathbb{R}$ its values. We are looking for an RBF interpolation function

$$
s(x)=\sum_{i=1}^{n} a_{i} \phi\left(x-x_{i}\right)+\sum_{j=1}^{m} b_{j} L_{j}(x),
$$

where $L_{j}$ for $j=1, \ldots, m$ are the Lagrange polynomials such that $L_{j}\left(x_{i}\right)=\delta_{i j}$ and $\phi$ a conditional positive definite RBF of order $m$. By assuming further that $m=n-1$, it holds

Lemma 3.4 (Explicit RBF solution formula). The interpolation problem (3.4) and (3.5) can be solved using an explicit formula if we choose an RBF interpolation ansatz with a conditional positive definite RBF of order smaller than $n-1$

$$
s(x)=\alpha \mathrm{d} \varphi(x)+\sum_{i=1}^{n-1} y_{i} L_{i}(x)
$$

where $\alpha=\frac{y_{N}-\sum_{i=1}^{n-1} y_{i} L_{i}\left(x_{n}\right)}{\mathrm{d} \varphi\left(x_{n}\right)}, \mathrm{d} \varphi(x)=\varphi(x)-\sum_{j=1}^{n-1} \varphi\left(x_{j}\right) L_{j}(x)$ and $\varphi(x)=\phi\left(x-x_{n}\right)-\sum_{i=1}^{n-1} L_{i}\left(x_{n}\right) \phi\left(x-x_{i}\right)$.

Proof. From the representation of the polynomial part using Lagrange polynomials we recover

$$
a_{j}=-a_{n} L_{j}\left(x_{n}\right), \quad \text { for } j=1, \ldots, n-1 .
$$

This yields the interpolation function

$$
s(x)=\alpha \varphi(x)+\sum_{j=1}^{n-1} b_{j} L_{j}(x),
$$

with $\alpha=a_{n}$, which solves the reduced interpolation problem

$$
\alpha \varphi\left(x_{i}\right)+\sum_{j=1}^{n-1} b_{j} L_{j}\left(x_{i}\right)=y_{i}, \quad \text { for } i=1, \ldots, n .
$$

By the properties of the Lagrange polynomials we recover the explicit form of $\alpha$ and $b_{j}$

$$
\begin{aligned}
\alpha & =\frac{y_{n}-\sum_{i=1}^{n-1} y_{i} L_{i}\left(x_{n}\right)}{\mathrm{d} \varphi\left(x_{n}\right)}, \\
b_{j} & =y_{j}-\alpha \varphi\left(x_{j}\right)
\end{aligned}
$$$$
\text { for } j=1, \ldots, n-1 \text {. }
$$ 
Remark 3.5. We can express $d \varphi$ in terms of projections

$$
\left.\mathrm{d} \varphi(x):=\Psi\left(x, x_{i}\right)=\left(I d-\mathcal{P}^{x}\right)\left(I d-\mathcal{P}^{y}\right)[\phi(x-y))\right]\left.\right|_{y=x_{i}},
$$

where the operators $\mathcal{P}^{z}$ is the projection of the variable $z$ on the polynomial space of dimension $n-1$. Schaback [35] shows that $\Psi$ is positive definite on $\mathbb{R}^{d} \backslash\left\{x_{1}, \ldots, x_{n-1}\right\}$. Thus, it is closely related to reproducing kernels and its native spaces, introduced in [35].

Remark 3.6. Note that this representation is independent of permutations of the indices. In general we can choose $\tilde{y}_{n}=y_{j}$ and $\tilde{y}_{i} \in\left\{y_{l} \mid l \neq j\right\}$.

\section{SMOOTHNESS INDiCATOR FOR RBF INTERPOLATION FUNCTIONS}

In essentially nonoscillatory (ENO)- and weighted ENO (WENO)-type methods a key component is to measure the smoothness of the interpolation function. In the polynomial ENO scheme, the highest degree divided difference plays an important role for identifying the least oscillating interpolation of a certain degree. To extend this to RBF-based interpolation we need something similar. However, the divided differences, used in the standard Newton's interpolation formula, are valid only for polynomials.

\subsection{Generalized divided differences}

For non-polynomial basis functions Mühlbach [29] introduces generalized divided differences, which coincide in the monomial case with the standard one. The result is based on functions $f_{1}, \ldots, f_{n}$ that form a Chebyshev system, i.e., they satisfy

$$
\left|\begin{array}{ccc}
f_{1}\left(z_{1}\right) & \cdots & f_{1}\left(z_{k}\right) \\
\vdots & & \vdots \\
f_{k}\left(z_{1}\right) & \cdots & f_{k}\left(z_{k}\right)
\end{array}\right| \neq 0
$$

for all distinct points $z_{1}, \ldots, z_{k}$ and for $k=0, \ldots, n$. Using Cramer's rule we recover that for any $f: \mathbb{R} \rightarrow \mathbb{R}$ and set of distinct points $x_{1}, \ldots, x_{n}$ there exists a unique linear combination

$$
\mathrm{p}_{n} f:=\mathrm{p} f\left[\begin{array}{c}
f_{1}, \ldots, f_{n} \\
x_{1}, \ldots, x_{n}
\end{array}\right],
$$

of $f_{1}, \ldots, f_{n}$ which satisfy the interpolation condition

$$
\mathrm{p}_{n} f\left(x_{i}\right)=f\left(x_{i}\right), \quad \text { for all } i=1, \ldots, n .
$$

Theorem 4.1 (Generalized Newton's interpolation formula [30]). Let $f_{1}, \ldots, f_{n}$ form a complete Chebyshev system. Then for any $f: \mathbb{R} \rightarrow \mathbb{R}$ and any subset $G_{n}=\left\{x_{1}, \ldots x_{n}\right\} \subset \mathbb{R}$ of cardinality $n$ it holds

$$
\mathrm{p} f\left[\begin{array}{c}
f_{1}, \ldots, f_{n} \\
x_{1}, \ldots, x_{n}
\end{array}\right]=\sum_{k=1}^{n}\left[\begin{array}{c}
f_{1}, \ldots, f_{k} \\
x_{1}, \ldots, x_{k}
\end{array} \mid f\right] \cdot g_{k},
$$

where

$$
\begin{aligned}
& g_{1}:=f_{1}, \\
& g_{k}:=r_{k-1} f_{k}, \quad \text { for } k=2, \ldots, n,
\end{aligned}
$$

with the interpolation error in the kth step

$$
r_{k} f:=\mathrm{r} f\left[\begin{array}{c}
f_{1}, \ldots, f_{k} \\
x_{1}, \ldots, x_{k}
\end{array}\right]:=f-\mathrm{p} f\left[\begin{array}{c}
f_{1}, \ldots, f_{k} \\
x_{1}, \ldots, x_{k}
\end{array}\right],
$$


and the recursively defined coefficients

$$
\left[\begin{array}{c}
f_{1}, \ldots, f_{k} \\
x_{1}, \ldots, x_{k}
\end{array} \mid f\right]:=\frac{\left[\begin{array}{c}
f_{1}, \ldots, f_{k-1} \mid \\
x_{2}, \ldots, x_{k}
\end{array} \mid f\right]-\left[\begin{array}{c}
f_{1}, \ldots, f_{k-1} \mid \\
x_{1}, \ldots, x_{k-1} \mid
\end{array}\right]}{\left[\begin{array}{c}
f_{1}, \ldots, f_{k-1} \\
x_{2}, \ldots, x_{k}
\end{array} \mid f_{k}\right]-\left[\begin{array}{c}
f_{1}, \ldots, f_{k-1} \mid \\
x_{1}, \ldots, x_{k-1}
\end{array} \mid f_{k}\right]}, \quad \text { for } k \geqslant 2,
$$

where

$$
\left[\begin{array}{c}
f_{1} \\
x_{j}
\end{array} \mid f\right]:=\frac{f\left(x_{j}\right)}{f_{1}\left(x_{j}\right)}
$$

Based on this we can express the generalized divided differences for the basis $\left\{1, x, \ldots, x^{N-2}, \varphi\right\}$ for $N \in \mathbb{N}$ and $\varphi$ from Lemma 3.4 to quantify the oscillations of the interpolation function. To distinguish between the Lagrange polynomials of different degree we write $L_{j}^{i, d}$ for the Lagrange polynomial of degree $d$ such that $L_{j}^{i, d}\left(x_{l}\right)=\delta_{l j}$ for $l \in\{i-d-1, \ldots, i-1\}$.

Theorem 4.2. Let the basis be given by $\left\{1, x, \ldots, x^{N-2}, \varphi\right\}$ for $N \in \mathbb{N}$ and $\varphi$ as defined in Lemma 3.4. We recover the generalized divided differences of the form

$$
\begin{aligned}
{\left[\begin{array}{c}
1 \\
x_{0}
\end{array} \mid f\right] } & =f\left(x_{0}\right)=y_{0}, \\
{\left[\begin{array}{c}
1, x, \ldots, x^{k} \\
x_{1}, x_{2}, \ldots, x_{k+1}
\end{array} \mid f\right] } & =\frac{y_{k+1}-\sum_{i=1}^{k} y_{i} L_{i}^{k+1, k-1}\left(x_{k+1}\right)}{\prod_{i=1}^{k}\left(x_{k+1}-x_{i}\right)}, \quad \text { for } k<N-1, \\
{\left[\begin{array}{c}
1, x, \ldots, x^{N-2}, \varphi \\
x_{1}, x_{2}, \ldots, x_{N-1}, x_{N}
\end{array} \mid f\right] } & =\frac{y_{N}-\sum_{i=1}^{N-1} y_{i} L_{i}^{N, N-2}\left(x_{N}\right)}{\varphi\left(x_{N}\right)-\sum_{i=1}^{N-1} \varphi\left(x_{i}\right) L_{i}^{N, N-2}\left(x_{N}\right)}
\end{aligned}
$$

By comparing this results with the RBF interpolation in Lemma 3.4, we observe that the last divided difference can be written as

$$
\left[\begin{array}{c}
1, x, \ldots, x^{N-2}, \varphi \\
x_{1}, x_{2}, \ldots, x_{N-1}, x_{N}
\end{array} \mid f\right]=\alpha .
$$

This suggests that $\alpha$ may be a good choice as the smoothness indicator based on the success of the classic ENO scheme.

\subsection{Relation to reproducing Kernel Hilbert spaces and its norm}

As mentioned above there is a close relation to native spaces of conditionally positive definite functions (see Schaback [35]). Indeed, the RBF-based basis function $\mathrm{d} \varphi$ can be expressed in terms of the modified kernel function $\left.\Psi(x, y)=\left(I d-\mathcal{P}^{x}\right)\left(I d-\mathcal{P}^{y}\right)[\phi(x-y))\right]$.

By analysing the norm of the interpolation function, based on the inner product of the native space, we have

Lemma 4.3. Let $s$ be a RBF-interpolation function given by (3.13). Then, it has the norm

$$
\|s\|_{\phi}^{2}=\sum_{i=1}^{N-1} s\left(x_{i}\right)^{2}+\alpha^{2} \mathrm{~d} \varphi\left(x_{N}\right) .
$$

In particular, we have

$$
\|s\|_{\phi} \approx \frac{\beta}{\mathrm{d} \varphi\left(x_{N}\right)^{1 / 2}}
$$

with $\beta=y_{N}-\sum_{i=1}^{N-1} y_{i} L_{i}\left(x_{N}\right)$. 
This lemma proposes a scaling of $\mathrm{d} \varphi\left(x_{N}\right)^{1 / 2}$ of our smoothness indicator.

Proof. The inner product of the native space is

$$
(f, g)_{\phi}=\sum_{i=1}^{N-1} f\left(x_{i}\right) g\left(x_{i}\right)+(f-\mathcal{P} f, g-\mathcal{P} g)_{\phi, 0},
$$

with

$$
(f, g)_{\phi, 0}=\sum_{j=1}^{M} \sum_{k=1}^{N} \lambda_{j} \mu_{k} \phi\left(x_{j}, y_{k}\right),
$$

for $f=\sum_{j=1}^{M} \lambda_{j} \phi\left(x, x_{j}\right)$ and $g=\sum_{k=1}^{N} \mu_{k} \phi\left(x, y_{k}\right)[35]$.

We have $(s-\mathcal{P} s)(x)=\beta \frac{\mathrm{d} \varphi(x)}{\mathrm{d} \varphi\left(x_{N}\right)}$ and

$$
\|s\|_{\phi}^{2}=\sum_{j=1}^{N-1} s\left(x_{j}\right)^{2}+\left(\frac{\beta}{\mathrm{d} \varphi\left(x_{N}\right)}\right)^{2}(\mathrm{~d} \varphi, \mathrm{d} \varphi)_{\phi} .
$$

Finally, we insert the definition of $\mathrm{d} \varphi$ to recover

$$
\begin{aligned}
(\mathrm{d} \varphi, \mathrm{d} \varphi)_{\phi} & =(\mathrm{d} \varphi, \mathrm{d} \varphi)_{\phi, 0}, \\
& =\phi(0)-2 \sum_{j=1}^{N-1} \phi\left(x_{N}-x_{j}\right) L_{j}\left(x_{N}\right)+\sum_{j, k=1}^{N-1} \phi\left(x_{k}-x_{j}\right) L_{j}\left(x_{N}\right) L_{k}\left(x_{N}\right), \\
& =\mathrm{d} \varphi\left(x_{N}\right) .
\end{aligned}
$$

Corollary 4.4. Let $\mathrm{d} \varphi$ be given as in Lemma 3.4. We have

$$
\mathrm{d} \varphi\left(x_{N}\right)>0 .
$$

Lemma 4.5 (Equivalent Norm). The set defined by

$$
\mathcal{B}:=\{\varphi\} \cup\left\{\frac{\varphi\left(x_{j}\right)}{L_{j}\left(x_{N}\right)} L_{j} \mid j=1, \ldots, N-1\right\},
$$

is a basis of the interpolation space. In particular, we have equivalence of the norms $\|\cdot\|_{\phi}$ and $\|\cdot\|_{\mathcal{B}}$, where

$$
\|s\|_{\mathcal{B}}^{2}=\sum_{i=1}^{N} \alpha_{i}^{2}
$$

for $s(x)=\alpha_{N} \varphi(x)+\sum_{i=1}^{N-1} \alpha_{i} \frac{\varphi\left(x_{j}\right)}{L_{j}\left(x_{N}\right)} L_{j}(x)$.

Proof. From the interpolation (3.13) we recover that $\mathcal{B}$ is a basis of the interpolation space.

\subsection{Smoothness indicator and stencil choice}

Harten et al. proposed the Essentially Nonoscillatory method to control spurious oscillations at discontinuities [18]. Its principle is based on the evaluation of multiple stencils for each cell $C_{i}$ in which we need to reconstruct the solution. Finally, one chooses the least oscillatory reconstruction to define $s_{i}$. Fjordholm et al. [12] showed the sign-property for the polynomial reconstruction method with the recursive algorithm introduced in [18] which utilizes the last divided difference as a local smoothness indicator. A sign preserving WENO reconstruction method was proposed by Fjordholm et al. [13]. In the RBF reconstruction, the highest derivative is similar to the RBF-part of the reconstruction in Lemma 4.3 and Theorem 4.2. As we shall show, the recursive algorithm from the polynomial case, combined with the smoothness indicator 


$$
\operatorname{IS}(s)=\frac{\beta}{\mathrm{d} \varphi\left(x_{N}\right)^{1 / 2}} \quad \text { with } \beta=y_{N}-\sum_{i=1}^{N-1} y_{i} L_{i}\left(x_{N}\right),
$$

is sign-stable for small enough grid sizes. Numerical experiments confirm this to be true for general grids. In the next section we prove this for the second and third degree reconstructions on general grids.

Note that Corollary 4.4 ensures the definition of IS $(s)$.

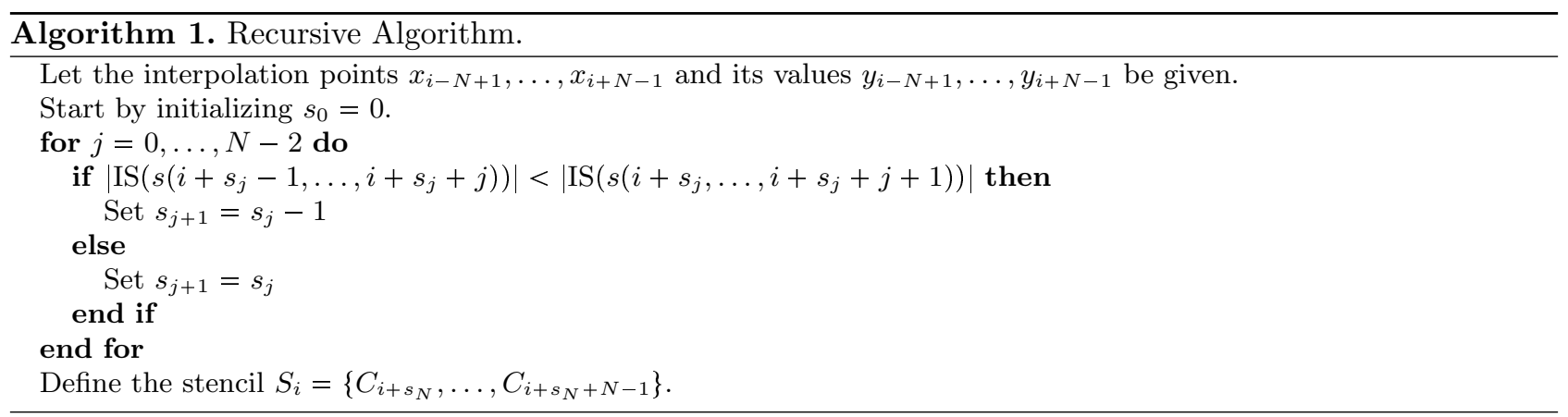

Remark 4.6. The restriction that the sign-property holds only on grids with small grid size is not a limitation. For infinitely smooth RBFs we can choose a small shape parameter to decrease the computational grid size.

Remark 4.7. The smoothness indicator (4.19) requires an impractical and computationally expensive evaluation. However, from Lemma 4.5 we recover

$$
\mathrm{d} \varphi\left(x_{N}\right)=\|\mathrm{d} \varphi\|_{\phi}^{2} \approx\|\mathrm{d} \varphi\|_{\mathcal{B}}^{2}=\left(1+\sum_{i=1}^{N-1} L_{i}\left(x_{N}\right)^{2}\right) .
$$

Thus, we have equivalence of the smoothness indicator IS with

$$
\widetilde{\mathrm{IS}}(s):=\frac{\beta}{\mathrm{d} \varphi\left(x_{N}\right)}\left(1+\sum_{i=1}^{N-1} L_{i}\left(x_{N}\right)^{2}\right)^{1 / 2}=\left(\sum_{i=1}^{N} a_{i}^{2}\right)^{1 / 2} .
$$

To choose the least oscillatory stencil $S_{i}$ for the $i$ th cell for the RBF-reconstruction we follow Algorithm 1 which is based on the one from Harten et al. [18]. We use the notation $s(j, \ldots, j+k)$ that corresponds to the reconstruction on the cells $C_{j}, \ldots, C_{j+k}$ with the interpolation points $x_{j}, \ldots, x_{j+k}$ and its values $y_{j}, \ldots, y_{j+k}$.

Remark 4.8. In the general case of $N \geqslant M+1$ and a conditionally positive definite RBF of order $M$, we replace $\alpha$ by $\sqrt{\sum_{i=1}^{N} a_{i}^{2}}$ in Algorithm 1. In this case it is more difficult to prove the sign-property, but numerical experiments suggest that it remains valid.

\section{Sign-PROPERTY FOR 2ND AND 3RD DEGREE RECONSTRUCTION}

Based on the results from the previous sections we show the sign-property of the RBF interpolation for the second and third degree reconstruction, i.e. $N=2,3$. This means that we deal with stencils $S_{i}$ of size $N$ which represent the interpolation points for the reconstruction on cell $C_{i}$. Let us name them

$$
\begin{aligned}
S_{i} & =\left\{C_{i+r_{N-1}}, \ldots, C_{i+r_{N-1}+N-1}\right\}, \\
S_{i+1} & =\left\{C_{i+s_{N-1}+1}, \ldots, C_{i+s_{N-1}+N}\right\},
\end{aligned}
$$

where $r_{N-1} \leqslant 1+s_{N-1}$ and $C_{j}$ is the $j$ th cell with its mid-point $x_{j}$ on which we apply the interpolation. Further, we define $d_{N-1}:=1+s_{N-1}-r_{N-1} \geqslant 0$ as the shift between the stencils. The stencils are chosen by Algorithm 1 and there are no constraints on the stencils. 


\subsection{Notation}

For simplicity, we introduce some general notation. We assume the stencil length to be $N$ and we name terms by the highest appearing index $j$ that exists in the underlying stencil $C_{j-N+1}, \ldots, C_{j}$. We also define

$$
\begin{aligned}
L_{j}^{i}(x)- & \text { Lagrange polynomial of degree } N-1 \text { such that } \\
& L_{j}^{i}\left(x_{l}\right)=\delta_{l j} \text { for } l \in\{i-N+1, \ldots, i-1\}, \\
\varphi^{j}(x):= & \phi\left(x-x_{j}\right)-\sum_{l=1}^{N-1} \phi\left(x-x_{j-N+l}\right) L_{j-N+l}^{j}\left(x_{j}\right), \\
\mathrm{d} \varphi^{j}(x):= & \varphi^{j}(x)-\sum_{i=1}^{N-1} \varphi^{j}\left(x_{i}\right) L_{i}^{j}(x), \\
\alpha_{j}:= & \frac{\beta_{j}}{\mathrm{~d} \varphi^{j}\left(x_{j}\right)}, \quad \gamma_{j}:=\frac{\beta_{j}}{\mathrm{~d} \varphi^{j}\left(x_{j}\right)^{1 / 2}}, \quad \beta_{j}:=y_{j}-\sum_{i=1}^{N-1} y_{j-N+i} L_{j-N+i}^{j}\left(x_{j}\right) .
\end{aligned}
$$

\subsection{Representation of the reconstructed jumps}

The idea of the proof is to give a simple representation of the reconstructed jumps

$$
\mathrm{jR}_{i+1 / 2}:=s_{i+1}\left(x_{i+1 / 2}\right)-s_{i}\left(x_{i+1 / 2}\right),
$$

and show that each term has the same sign as the jump in its neighboring cells. Let us assume that we have given the stencils $S_{i}$ and $S_{i+1}$ for the cells $i$ and $i+1$ from Algorithm 1.

Theorem 5.1 (Generalized representation). The second and third degree reconstructed jump can be written in the following form

$$
\mathrm{jR}_{i+1 / 2}=\sum_{j=0}^{d_{N-1}-1} C_{j}\left(\gamma_{i+r_{N-1}+N+j}-\gamma_{i+r_{N-1}+N-1+j}\right)+\varepsilon(\Delta x),
$$

with the constants

$$
\begin{aligned}
& C_{0}=\frac{\mathrm{d} \varphi^{k}\left(x_{i+1 / 2}\right)}{\delta_{k}}-A_{k} \delta_{k} \\
& C_{j}=C_{j-1}-A_{k+j} \delta_{k+j}=\frac{\mathrm{d} \varphi^{k}\left(x_{i+1 / 2}\right)}{\delta_{k}}-\sum_{l=0}^{j} A_{k+l} \delta_{k+l},
\end{aligned}
$$

and an error term

$$
\varepsilon(\Delta x)=\gamma_{k+d_{N-1}}\left(\frac{\mathrm{d} \varphi^{k+d_{N-1}}\left(x_{i+1 / 2}\right)}{\delta_{k+d_{N-1}}}-C_{d_{N-1}-1}\right),
$$

where $k=i+r_{N-1}+N-1$.

The proof relies on multiple Lemmas which we now develop.

Lemma 5.2. Given the Lagrange polynomials. For $N=2,3$ it holds

$$
-\sum_{l=1}^{N-1} y_{j-N+l} L_{j-N+l}^{j}\left(x_{i+1 / 2}\right)=A_{j} \beta_{j}-\sum_{l=1}^{N-1} y_{j-N+l+1} L_{j-N+l+1}^{j+1}\left(x_{i+1 / 2}\right),
$$

where

$$
A_{j}=\frac{L_{j-N+1}^{j}\left(x_{i+1 / 2}\right)}{L_{j-N+1}^{j}\left(x_{j}\right)}
$$


Proof. The case $N=2$ is immediate since the Lagrange polynomials are constant. Thus, (5.10) is

$$
-y_{j-1}=\beta_{j}-y_{j},
$$

For $N=3$, we write the left hand side of (5.10) and subtract $A_{j} \beta_{j}$

$$
-y_{j-2} L_{j-2}^{j}\left(x_{i+1 / 2}\right)-y_{j-1} L_{j-1}^{j}\left(x_{i+1 / 2}\right)-A_{j} \beta_{j}=-y_{j-1}\left(L_{j-1}^{j}\left(x_{i+1 / 2}\right)-A_{j} L_{j-1}^{j}\left(x_{j}\right)\right)-y_{j} A_{j} .
$$

Note that $A_{j}=L_{j}^{j+1}\left(x_{i+1 / 2}\right)$ and consider

$$
A_{j} L_{j-1}^{j}\left(x_{j}\right)-L_{j-1}^{j}\left(x_{i+1 / 2}\right)=\frac{\left(x_{i+1 / 2}-x_{j}\right)}{\left(x_{j}-x_{j-1}\right)}=-L_{j-1}^{j+1}\left(x_{i+1 / 2}\right) .
$$

Lemma 5.3. The reconstructed jump $j R_{i+1 / 2}$ for the second and third degree reconstruction method can be expressed as

$$
\mathrm{j} \mathrm{R}_{i+1 / 2}=\frac{\gamma_{k+d_{N-1}}}{\delta_{k+d_{N-1}}} \mathrm{~d} \varphi^{k+d_{N-1}}\left(x_{i+1 / 2}\right)-\frac{\gamma_{k}}{\delta_{k}} \mathrm{~d} \varphi^{k}\left(x_{i+1 / 2}\right)+\sum_{j=0}^{d_{N-1}-1} A_{k+j} \gamma_{k+j} \delta_{k+j},
$$

where $k=i+r_{N-1}+N-1, k+d_{N-1}=i+s_{N-1}+N$ and $\delta_{i}=\mathrm{d} \varphi^{i}\left(x_{i}\right)^{1 / 2}$.

Proof. From Lemma 3.4 and the stencils selected from (5.1) we rewrite the $N$ th degree reconstructed jump $\mathrm{jR}_{i+1 / 2}$ between cell $i$ and $i+1$ as

$$
\begin{aligned}
\mathrm{j} \mathrm{R}_{i+1 / 2}= & \alpha_{i+s_{N-1}+N} \mathrm{~d} \varphi^{i+s_{N-1}+N}\left(x_{i+1 / 2}\right)-\alpha_{i+r_{N-1}+N-1} \mathrm{~d} \varphi^{i+r_{N-1}+N-1}\left(x_{i+1 / 2}\right) \\
& +\sum_{j=1}^{N-1} y_{i+s_{N-1}+j} L_{i+s_{N-1}+j}^{i+s_{N-1}+N}\left(x_{i+1 / 2}\right)-\sum_{j=1}^{N-1} y_{i+r_{N-1}+j-1} L_{i+r_{N-1}+j-1}^{i+r_{N-1}+N-1}\left(x_{i+1 / 2}\right) .
\end{aligned}
$$

The polynomial part of the reconstructed jump is

$$
p_{i+1}\left(x_{i+1 / 2}\right)-p_{i}\left(x_{i+1 / 2}\right)=\sum_{j=0}^{d_{N-1}-1} A_{i+r_{N-1}+N-1+j} \beta_{i+r_{N-1}+N-1+j},
$$

by recursively applying Lemma 5.2. This yields

$$
\mathrm{jR}_{i+1 / 2}=\alpha_{k+d_{N-1}} \mathrm{~d} \varphi^{k+d_{N-1}}\left(x_{i+1 / 2}\right)-\alpha_{k} \mathrm{~d} \varphi^{k}\left(x_{i+1 / 2}\right)+\sum_{j=0}^{d_{N-1}-1} A_{k+j} \mathrm{~d} \varphi^{k+j}\left(x_{k+j}\right) \alpha_{k+j} .
$$

By inserting $\gamma_{i}=\alpha_{i} \mathrm{~d} \varphi^{i}\left(x_{i}\right)^{1 / 2}$ we recover the result.

Lemma 5.4. We have

$$
A_{j} \mathrm{~d} \varphi^{j}\left(x_{j}\right)-\mathrm{d} \varphi^{j}\left(x_{i+1 / 2}\right)=-\left(\varphi^{j}-\mathcal{P}_{j+1}^{N-1} \varphi^{j}\right)\left(x_{i+1 / 2}\right),
$$

with $\mathcal{P}_{j+1}^{k}$ as the $k$ th degree polynomial approximation with respect to the interpolation points $x_{j}, \ldots, x_{j+1-k}$.

Proof. In the case $N=2$, we have $A_{j}=1$ and $L_{j}=1$ and recover

$$
\begin{aligned}
\mathrm{d} \varphi^{j}\left(x_{j}\right)-\mathrm{d} \varphi^{j}\left(x_{i+1 / 2}\right) & =\phi(0)-\phi\left(x_{j}-x_{j-1}\right)-\phi\left(x_{i+1 / 2}-x_{j}\right)+\phi\left(x_{i+1 / 2}-x_{j-1}\right), \\
& =-\left(\varphi^{j}-\mathcal{P}_{j+1}^{1} \varphi^{j}\right)\left(x_{i+1 / 2}\right) .
\end{aligned}
$$


In the case $N=3$, we have

$$
\begin{aligned}
& A_{j} \mathrm{~d} \varphi^{j}\left(x_{j}\right)-\mathrm{d} \varphi^{j}\left(x_{i+1 / 2}\right)=\left(\varphi^{j}\left(x_{j}\right) L_{j-2}^{j}\left(x_{i+1 / 2}\right)-\varphi^{j}\left(x_{i+1 / 2}\right) L_{j-2}^{j}\left(x_{j}\right)\right. \\
& -\varphi^{j}\left(x_{j-1}\right) L_{j-1}^{j}\left(x_{j}\right) L_{j-2}^{j}\left(x_{i+1 / 2}\right)-\varphi^{j}\left(x_{j-2}\right) L_{j-2}^{j}\left(x_{j}\right) L_{j-2}^{j}\left(x_{i+1 / 2}\right) \\
& \left.+\varphi^{j}\left(x_{j-1}\right) L_{j-1}^{j}\left(x_{i+1 / 2}\right) L_{j-2}^{j}\left(x_{j}\right)+\varphi^{j}\left(x_{j-2}\right) L_{j-2}^{j}\left(x_{i+1 / 2}\right) L_{j-2}^{j}\left(x_{j}\right)\right) \frac{1}{L_{j-2}^{j}\left(x_{j}\right)},
\end{aligned}
$$

which can be simplified as

$$
\begin{aligned}
A_{j} \mathrm{~d} \varphi^{j}\left(x_{j}\right)-\mathrm{d} \varphi^{j}\left(x_{i+1 / 2}\right)= & \left(\varphi^{j}\left(x_{j}\right) L_{j-2}^{j}\left(x_{i+1 / 2}\right)-\varphi^{j}\left(x_{i+1 / 2}\right) L_{j-2}^{j}\left(x_{j}\right)\right. \\
& \left.+\varphi^{j}\left(x_{j-1}\right)\left(L_{j-1}^{j}\left(x_{i+1 / 2}\right) L_{j-2}^{j}\left(x_{j}\right)-L_{j-1}^{j}\left(x_{j}\right) L_{j-2}^{j}\left(x_{i+1 / 2}\right)\right)\right) \frac{1}{L_{j-2}^{j}\left(x_{j}\right)} .
\end{aligned}
$$

Next, we express the last term

$$
L_{j-1}^{j}\left(x_{i+1 / 2}\right) L_{j-2}^{j}\left(x_{j}\right)-L_{j-1}^{j}\left(x_{j}\right) L_{j-2}^{j}\left(x_{i+1 / 2}\right)=L_{j-2}^{j}\left(x_{j}\right)-L_{j-2}^{j}\left(x_{i+1 / 2}\right),
$$

and insert this into $(5.21)$

$$
\begin{aligned}
A_{j} \mathrm{~d} \varphi^{j}\left(x_{j}\right)-\mathrm{d} \varphi^{j}\left(x_{i+1 / 2}\right) & =-\varphi^{j}\left(x_{i+1 / 2}\right)+\varphi^{j}\left(x_{j}\right) L_{j}^{j+1}\left(x_{i+1 / 2}\right)+\varphi^{j}\left(x_{j-1}\right) L_{j-1}^{j+1}\left(x_{i+1 / 2}\right), \\
& =-\left(\varphi^{j}-\mathcal{P}_{j+1}^{N-1} \varphi^{j}\right)\left(x_{i+1 / 2}\right) .
\end{aligned}
$$

where we use that

$$
L_{j}^{j+1}\left(x_{i+1 / 2}\right)=\frac{L_{j-2}^{j}\left(x_{i+1 / 2}\right)}{L_{j-2}^{j}\left(x_{j}\right)}, \quad L_{j-1}^{j+1}\left(x_{i+1 / 2}\right)=1-\frac{L_{j-2}^{j}\left(x_{i+1 / 2}\right)}{L_{j-2}^{j}\left(x_{j}\right)} .
$$

Now, we are ready to prove Theorem 5.1.

Proof. (Thm. 5.1)

The goal is to show the equivalence with the representation in Lemma 5.3. Therefore, we insert (5.9) into (5.7) to recover

$$
\begin{aligned}
j R_{i+1 / 2}= & C_{d_{N-1}-1} \gamma_{k+d_{N-1}}+\sum_{j=1}^{d_{N-1}-1} \gamma_{k+j}\left(C_{j-1}-C_{j}\right)-C_{0} \gamma_{k}+\varepsilon(\Delta x), \\
= & C_{d_{N-1}-1} \gamma_{k+d_{N-1}}+\sum_{j=1}^{d_{N-1}-1} \gamma_{k+j}\left(C_{j-1}-C_{j}\right)+A_{k} \delta_{k} \gamma_{k} \\
& -\frac{\gamma_{k}}{\delta_{k}} \mathrm{~d} \varphi^{k}\left(x_{i+1 / 2}\right)+\gamma_{k+d_{N-1}}\left(\frac{\mathrm{d} \varphi^{k+d_{N-1}}\left(x_{i+1 / 2}\right)}{\delta_{k+d_{N-1}}}-C_{d_{N-1}-1}\right) .
\end{aligned}
$$

Finally, we insert the definition of $C_{j}$ to obtain

$$
j R_{i+1 / 2}=\frac{\gamma_{k+d_{N-1}}}{\delta_{k+d_{N-1}}} \mathrm{~d} \varphi^{k+d_{N-1}}\left(x_{i+1 / 2}\right)+\sum_{j=0}^{d_{N-1}-1} A_{k+j} \delta_{k+j} \gamma_{k+j}-\frac{\gamma_{k}}{\delta_{k}} \mathrm{~d} \varphi^{k}\left(x_{i+1 / 2}\right) .
$$


Remark 5.5. Let us define

$$
\varepsilon_{j}(\Delta x):=\frac{1}{\delta_{j+1} \delta_{j}}\left(\mathrm{~d} \varphi^{j+1}\left(x_{i+1 / 2}\right) \frac{\delta_{j}}{\delta_{j+1}}-\mathrm{d} \varphi^{j}\left(x_{i+1 / 2}\right)+A_{j} \mathrm{~d} \varphi^{j}\left(x_{j}\right)\right) .
$$

Thus, the error $\varepsilon(\Delta x)$ can be written as

$$
\varepsilon(\Delta x)=\beta_{k+d} \sum_{i=0}^{d_{N-1}} \varepsilon_{k+i}(\Delta x) \frac{\delta_{k+i+1}}{\delta_{k+d}} .
$$

From Lemma 5.4 we can express $\varepsilon_{j}(\Delta x)$ by

$$
\varepsilon_{j}(\Delta x)=\frac{1}{\delta_{j+1} \delta_{j}}\left(\mathrm{~d} \varphi^{j+1}\left(x_{i+1 / 2}\right) \frac{\delta_{j}}{\delta_{j+1}}-\left(\varphi^{j}-\mathcal{P}_{j+1}^{N-1} \varphi^{j}\right)\left(x_{i+1 / 2}\right)\right) .
$$

\subsection{Sign-property for small grid size}

In this section we analyse the reconstructed jumps for infinitely smooth RBFs for small grid size $\Delta x \rightarrow 0$. From Theorem 5.1 we have a simple expression for the reconstructed jump. We first show that the error $\varepsilon(\Delta x)$ goes to zero, as the grid size goes to zero. Then, we show that each term of the remaining equation has the sign of the jump $y_{i+1}-y_{i}$.

Remark 5.6. The notation $\mathcal{O}\left(\Delta x^{p}\right)$ for $\Delta x \rightarrow 0$ should be interpreted in the way that given a grid $\hat{x}_{0}<\hat{x}_{1}<$ $\cdots<\hat{x}_{m}$ we analyse the terms for the grid $x_{0}<x_{1}<\cdots<x_{m}$ with $x_{j}=\hat{x}_{j} \Delta x$ and we use

$$
\mathcal{O}\left(\Delta x^{p}\right) \leqslant C \Delta x^{p}, \quad \text { for } \Delta x \rightarrow 0 .
$$

Remark 5.7. When calculating the errors $\varepsilon_{j}$ we recall that

$$
\mathrm{d} \varphi^{j}(x)=\varphi^{j}(x)-\mathcal{P}_{j}^{N-1} \varphi^{j}(x) .
$$

Theorem 5.8. Let $\phi$ be an infinitely smooth RBF of first or second order. Then, we have that $\varepsilon(\Delta x)=\mathcal{O}\left(\Delta x^{2}\right)$ for $\Delta x \rightarrow 0$ for $N=2,3$ and

$$
\mathrm{jR}_{i+1 / 2}=\sum_{j=0}^{d_{N-1}-1} C_{j}\left(\gamma_{i+r_{N-1}+N+j}-\gamma_{i+r_{N-1}+N-1+j}\right)+\mathcal{O}\left(\Delta x^{2}\right) .
$$

Proof. We start by analysing the different parts in the error term $\varepsilon_{k}(\Delta x)$. Note that as $\phi$ is a conditionally positive definite RBF

$$
\phi(x)=h\left(x^{2}\right) .
$$

Thus, it follows by induction that $\phi^{(2 k+1)}(0)=0$ for $k \in \mathbb{N}$ and we can neglect odd terms in the Taylor expansions.

Let us start with the case $N=2$ and a first order RBF:

$$
\begin{aligned}
\mathrm{d} \varphi^{k}(y) & =\phi\left(y-x_{k}\right)-\phi\left(y-x_{k-1}\right)-\phi\left(x_{k-1}-x_{k}\right)+\phi(0), \\
& =\frac{\phi^{\prime \prime}(0)}{2}\left(\left(y-x_{k}\right)^{2}-\left(y-x_{k-1}\right)^{2}-\left(x_{k-1}-x_{k}\right)^{2}\right)+\mathcal{O}\left(\Delta x^{4}\right), \\
& =-\phi^{\prime \prime}(0)\left(x_{k-1}-x_{k}\right)\left(x_{k-1}-y\right)+\mathcal{O}\left(\Delta x^{4}\right) .
\end{aligned}
$$

We further have that

$$
\begin{aligned}
\left(\varphi^{k}-\mathcal{P}_{k+1}^{1} \varphi^{k}\right)(y) & =\phi\left(y-x_{k}\right)-\phi\left(y-x_{k-1}\right)-\phi(0)+\phi\left(x_{k}-x_{k-1}\right) \\
& =\frac{\phi^{\prime \prime}(0)}{2}\left(\left(y-x_{k}\right)^{2}-\left(y-x_{k-1}\right)^{2}+\left(x_{k-1}-x_{k}\right)^{2}\right)+\mathcal{O}\left(\Delta x^{4}\right), \\
& =-\phi^{\prime \prime}(0)\left(x_{k-1}-x_{k}\right)\left(x_{k}-y\right)+\mathcal{O}\left(\Delta x^{4}\right) .
\end{aligned}
$$


From (5.32) we recover

$$
\begin{aligned}
\frac{\delta_{k}}{\delta_{k+1}} & =\frac{x_{k}-x_{k-1}}{x_{k+1}-x_{k}}+\mathcal{O}\left(\Delta x^{2}\right), \\
\delta_{k} \delta_{k+1} & =\frac{\phi^{\prime \prime}(0)}{2}\left(x_{k}-x_{k-1}\right)\left(x_{k+1}-x_{k}\right)+\mathcal{O}\left(\Delta x^{4}\right)=\mathcal{O}\left(\Delta x^{2}\right),
\end{aligned}
$$

which allows us to conclude

$$
\varepsilon_{k}(\Delta x)=\mathcal{O}\left(\Delta x^{2}\right)
$$

To find a bound for $\varepsilon(\Delta x)$ we further need $\delta_{k} / \delta_{k+1}=\mathcal{O}(1)$ and $\beta_{k+d}=\mathcal{O}(1)$. The latter is clear since the reconstructed function is bounded and $L_{j}^{i}\left(x^{i}\right)$ is $\mathcal{O}(1)$. Further, $\delta_{k} / \delta_{k+1}=\mathcal{O}(1)$ results from (5.34). Using (5.36) in (5.26) we conclude that $\varepsilon(\Delta x)=\mathcal{O}\left(\Delta x^{2}\right)$.

Next, we consider the more complicated case with $N=3$ and a second order RBF $\phi$. Hence, we need to analyse the following two terms:

$$
\begin{aligned}
\mathrm{d} \varphi^{k+1}(y)= & \varphi^{k+1}(y)-\varphi^{k+1}\left(x_{k}\right) L_{k}^{k+1}(y)-\varphi^{k+1}\left(x_{k-1}\right) L_{k-1}^{k+1}(y) \\
= & \phi\left(y-x_{k+1}\right)-\phi\left(y-x_{k}\right) L_{k}^{k+1}\left(x_{k+1}\right)-\phi\left(y-x_{k-1}\right) L_{k-1}^{k+1}\left(x_{k+1}\right) \\
& -\left(\phi\left(x_{k}-x_{k+1}\right)-\phi(0) L_{k}^{k+1}\left(x_{k+1}\right)-\phi\left(x_{k}-x_{k-1}\right) L_{k-1}^{k+1}\left(x_{k+1}\right)\right) L_{k}^{k+1}(y) \\
& -\left(\phi\left(x_{k-1}-x_{k+1}\right)-\phi\left(x_{k-1}-x_{k}\right) L_{k}^{k+1}\left(x_{k+1}\right)-\phi(0) L_{k-1}^{k+1}\left(x_{k+1}\right)\right) L_{k-1}^{k+1}(y) \\
\left(\varphi^{k}-\mathcal{P}_{k+1}^{2} \varphi^{k}\right)(y)= & \varphi^{k}(y)-\varphi^{k}\left(x_{k}\right) L_{k}^{k+1}(y)-\varphi^{k}\left(x_{k-1}\right) L_{k-1}^{k+1}(y) \\
= & \phi\left(y-x_{k}\right)-\phi\left(y-x_{k-1}\right) L_{k-1}^{k}\left(x_{k}\right)-\phi\left(y-x_{k-2}\right) L_{k-2}^{k}\left(x_{k}\right) \\
& -\left(\phi(0)-\phi\left(x_{k}-x_{k-1}\right) L_{k-1}^{k}\left(x_{k}\right)-\phi\left(x_{k}-x_{k-2}\right) L_{k-2}^{k}\left(x_{k}\right)\right) L_{k}^{k+1}(y) \\
& -\left(\phi\left(x_{k-1}-x_{k}\right)-\phi(0) L_{k-1}^{k}\left(x_{k}\right)-\phi\left(x_{k-1}-x_{k-2}\right) L_{k-2}^{k}\left(x_{k}\right)\right) L_{k-1}^{k+1}(y)
\end{aligned}
$$

As before, we apply the Taylor expansion

$$
\begin{aligned}
\varphi^{k+1}(y)= & \phi\left(y-x_{k+1}\right)-\phi\left(y-x_{k}\right) L_{k}^{k+1}\left(x_{k+1}\right)-\phi\left(y-x_{k-1}\right) L_{k-1}^{k+1}\left(x_{k+1}\right), \\
= & \frac{\phi^{\prime \prime}(0)}{2}\left(\left(y-x_{k+1}\right)^{2}-\left(y-x_{k}\right)^{2} L_{k}^{k+1}\left(x_{k+1}\right)-\left(y-x_{k-1}\right)^{2} L_{k-1}^{k+1}\left(x_{k+1}\right)\right) \\
& +\frac{\phi^{(4)}(0)}{2}\left(\left(y-x_{k}\right)^{4}-\left(y-x_{k}\right)^{4} L_{k}^{k+1}\left(x_{k+1}\right)-\left(y-x_{k-1}\right)^{4} L_{k-1}^{k+1}\left(x_{k+1}\right)\right) \\
& +\mathcal{O}\left(\Delta x^{6}\right) .
\end{aligned}
$$

We write

$$
\begin{array}{r}
\mathrm{d} \varphi^{k+1}(y)=a_{1} \frac{\phi^{\prime \prime}(0)}{2}+a_{2} \frac{\phi^{(4)}(0)}{4 !}+\mathcal{O}\left(\Delta x^{6}\right), \\
\left(\varphi^{k}-\mathcal{P}_{k+1}^{2} \varphi^{k}\right)(y)=b_{1} \frac{\phi^{\prime \prime}(0)}{2}+b_{2} \frac{\phi^{(4)}(0)}{4 !}+\mathcal{O}\left(\Delta x^{6}\right) .
\end{array}
$$

Let us calculate the coefficients $a_{1}$ and $a_{2}$

$$
a_{1}=a_{1}^{k-1} L_{k-1}^{k+1}\left(x_{k+1}\right)+a_{1}^{k} L_{k}^{k+1}\left(x_{k+1}\right) .
$$


From standard algebra we recover that $a_{1}^{k-1}=a_{1}^{k}=0$. The fourth order term is

$$
\begin{aligned}
a_{2}= & \left(y-x_{k+1}\right)^{4}-\left(y-x_{k}\right)^{4} L_{k}^{k+1}\left(x_{k+1}\right)-\left(y-x_{k-1}\right)^{4} L_{k-1}^{k+1}\left(x_{k+1}\right) \\
& -\left(\left(x_{k}-x_{k+1}\right)^{4}-\left(x_{k}-x_{k-1}\right)^{4} L_{k-1}^{k+1}\left(x_{k+1}\right)\right) L_{k}^{k+1}(y) \\
& -\left(\left(x_{k-1}-x_{k+1}\right)^{4}-\left(x_{k-1}-x_{k}\right)^{4} L_{k}^{k+1}\left(x_{k+1}\right)\right) L_{k-1}^{k+1}(y), \\
= & 6\left(x_{k-1}-x_{k+1}\right)\left(x_{k}-x_{k+1}\right)\left(x_{k-1}-y\right)\left(x_{k}-y\right) .
\end{aligned}
$$

We repeat this for the coefficients $b_{1}$ and $b_{2}$

$$
b_{1}=b_{1}^{k-1} L_{k-1}^{k}\left(x_{k}\right)+b_{1}^{k-2} L_{k-2}^{k}\left(x_{k}\right),
$$

with $b_{1}^{k-1}=b_{1}^{k-2}=0$. The fourth order term is

$$
\begin{aligned}
b_{2}= & \left(y-x_{k}\right)^{4}-\left(y-x_{k-1}\right)^{4} L_{k-1}^{k}\left(x_{k}\right)-\left(y-x_{k-2}\right)^{4} L_{k-2}^{k}\left(x_{k}\right) \\
& -\left(-\left(x_{k}-x_{k-1}\right)^{4} L_{k-1}^{k}\left(x_{k}\right)-\left(x_{k}-x_{k-2}\right)^{4} L_{k-2}^{k}\left(x_{k}\right)\right) L_{k}^{k+1}(y) \\
& -\left(\left(x_{k-1}-x_{k}\right)^{4}-\left(x_{k-1}-x_{k-2}\right)^{4} L_{k-2}^{k}\left(x_{k}\right)\right) L_{k-1}^{k+1}(y), \\
= & 6\left(x_{k-2}-x_{k}\right)\left(x_{k-1}-x_{k}\right)\left(x_{k-1}-y\right)\left(x_{k}-y\right) .
\end{aligned}
$$

The results can be summarized as

$$
\begin{array}{r}
\mathrm{d} \varphi^{k+1}(y)=\frac{\phi^{(4)}(0)}{4}\left(x_{k-1}-x_{k+1}\right)\left(x_{k}-x_{k+1}\right)\left(x_{k-1}-y\right)\left(x_{k}-y\right)+\mathcal{O}\left(\Delta x^{6}\right), \\
\left(\varphi^{k}-\mathcal{P}_{k+1}^{2} \varphi^{k}\right)(y)=\frac{\phi^{(4)}(0)}{4}\left(x_{k-2}-x_{k}\right)\left(x_{k-1}-x_{k}\right)\left(x_{k-1}-y\right)\left(x_{k}-y\right)+\mathcal{O}\left(\Delta x^{6}\right) .
\end{array}
$$

From this we recover

$$
\begin{aligned}
\frac{\delta_{k}}{\delta_{k+1}} & =\frac{\left(x_{k}-x_{k-1}\right)\left(x_{k}-x_{k-2}\right)}{\left(x_{k+1}-x_{k}\right)\left(x_{k+1}-x_{x}\right)}+\mathcal{O}\left(\Delta x^{2}\right), \\
\delta_{k} \delta_{k+1} & =\frac{\phi^{(4)}(0)}{4}\left(x_{k}-x_{k-1}\right)\left(x_{k}-x_{k-2}\right)\left(x_{k+1}-x_{k}\right)\left(x_{k+1}-x_{x}\right)+\mathcal{O}\left(\Delta x^{6}\right), \\
& =\mathcal{O}\left(\Delta x^{4}\right) .
\end{aligned}
$$

Thus, we have

$$
\mathrm{d} \varphi^{k+1}(y) \frac{\delta_{k}}{\delta_{k+1}}-\left(\varphi^{k}-\mathcal{P}_{k+1}^{2} \varphi^{k}\right)(y)=\mathcal{O}\left(\Delta x^{6}\right)
$$

which yields

$$
\varepsilon_{k}(\Delta x)=\mathcal{O}\left(\Delta x^{2}\right)
$$

for $\Delta x \rightarrow 0$. Equivalent to the case $N=2$, we can combine (5.46) and (5.50) in (5.26) and conclude $\varepsilon(\Delta x)=$ $\mathcal{O}\left(\Delta x^{2}\right)$

Since the error term $\varepsilon(\Delta x)$ vanishes, the remaining step is to prove that each term of (5.30) has the same sign as the jump.

Theorem 5.9 (Sign-property of second and third degree RBF-reconstruction). Let us assume that the stencil $S_{i}$ and $S_{i+1}$ are chosen with the Algorithm 1. Then, for infinitely smooth RBFs of first or second order it holds that

$$
\operatorname{sgn}\left(C_{j}\left(\gamma_{i+r_{N-1}+N+j}-\gamma_{i+r_{N-1}+N-1+j}\right)\right)=\operatorname{sgn}\left(y_{i+1}-y_{i}\right),
$$

for all $j=0, \ldots, d_{N-1}-1$ and for $\Delta x \rightarrow 0$. 
Proof. The proof is based on a study of all possible choices of stencils, that may result from Algorithm 1:

- $S_{i}=\left\{C_{i-1}, C_{i}\right\}, S_{i+1}=\left\{C_{i}, C_{i+1}\right\}$,

- $S_{i}=\left\{C_{i-1}, C_{i}\right\}, S_{i+1}=\left\{C_{i+1}, C_{i+2}\right\}$,

- $S_{i}=\left\{C_{i}, C_{i+1}\right\}, S_{i+1}=\left\{C_{i+1}, C_{i+2}\right\}$,

$-\ldots$

For each case we look at any inequality due to Algorithm 1 to recover the particular stencil configuration, and show for each case that (5.51) is fulfilled.

Note that $j R_{i+1 / 2}=0$, if $S_{i}=S_{i+1}$. Hence, we do not include such cases in the analysis. Further, we use the notation

$$
A \approx B \quad \text { if } A=B+\mathcal{O}(\Delta x), \quad \text { for } \Delta x \rightarrow 0 .
$$

Let us first consider $N=2$ and assume $\phi$ is of first order.

Case 1. Consider the stencils $S_{i}=\left\{C_{i-1}, C_{i}\right\}, S_{i+1}=\left\{C_{i}, C_{i+1}\right\}$, which require the following conditions

$$
\left|\gamma_{i}\right|<\left|\gamma_{i+1}\right|, \quad\left|\gamma_{i+1}\right|<\left|\gamma_{i+2}\right|
$$

Further, we have the representation of the jump for small grid sizes

$$
j R_{i+1 / 2} \approx C_{0}\left(\gamma_{i+1}-\gamma_{i}\right)
$$

and from (5.32) it follows that

$$
C_{0}=\delta_{i}\left(\frac{\mathrm{d} \varphi^{i}\left(x_{i+1 / 2}\right)}{\mathrm{d} \varphi^{i}\left(x_{i}\right)}-1\right) \approx \delta_{i} \frac{x_{i+1 / 2}-x_{i}}{x_{i}-x_{i-1}}>0 .
$$

Hence

$$
\operatorname{sgn}\left(C_{0}\left(\gamma_{i+1}-\gamma_{i}\right)\right)=\operatorname{sgn}\left(\gamma_{i+1}-\gamma_{i}\right)=\operatorname{sgn}\left(\gamma_{i+1}\right)=\operatorname{sgn}\left(y_{i+1}-y_{i}\right),
$$

since

$$
|a|>|b| \Rightarrow \operatorname{sgn}(a-b)=\operatorname{sgn}(a) .
$$

Case 2. Consider the stencils $S_{i}=\left\{C_{i-1}, C_{i}\right\}, S_{i+1}=\left\{C_{i+1}, C_{i+2}\right\}$, which is equivalent to the conditions

$$
\left|\gamma_{i}\right|<\left|\gamma_{i+1}\right|, \quad\left|\gamma_{i+1}\right|>\left|\gamma_{i+2}\right| \text {. }
$$

The jump can be represented as

$$
j R_{i+1 / 2} \approx C_{0}\left(\gamma_{i+1}-\gamma_{i}\right)+C_{1}\left(\gamma_{i+2}-\gamma_{i+1}\right) .
$$

As before it holds that $\operatorname{sgn}\left(C_{0}\left(\gamma_{i+1}-\gamma_{i}\right)\right)=\operatorname{sgn}\left(y_{i+1}-y_{i}\right)$ and

$$
C_{1}=C_{0}-\delta_{i+1} \approx \delta_{i} \frac{x_{i+1 / 2}-x_{i+1}}{x_{i}-x_{i-1}}<0 .
$$

Thus, we get for the second term

$$
\operatorname{sgn}\left(C_{1}\left(\gamma_{i+2}-\gamma_{i+1}\right)\right)=\operatorname{sgn}\left(\gamma_{i+1}-\gamma_{i+2}\right)=\operatorname{sgn}\left(\gamma_{i+1}\right)=\operatorname{sgn}\left(y_{i+1}-y_{i}\right),
$$

where we used (5.55) and (5.56). 
Case 3. In the last case of the second degree reconstruction we have the stencils $S_{i}=\left\{C_{i}, C_{i+1}\right\}, S_{i+1}=$ $\left\{C_{i+1}, C_{i+2}\right\}$, equivalent to the conditions

$$
\left|\gamma_{i}\right|>\left|\gamma_{i+1}\right|, \quad\left|\gamma_{i+1}\right|>\left|\gamma_{i+2}\right|
$$

The representation of the jump is

$$
j R_{i+1 / 2} \approx C_{0}\left(\gamma_{i+2}-\gamma_{i+1}\right) .
$$

As in the first case we recover from (5.32), that

$$
C_{0}=\delta_{i+1}\left(\frac{\mathrm{d} \varphi^{i+1}\left(x_{i+1 / 2}\right)}{\mathrm{d} \varphi^{i+1}\left(x_{i}\right)}-1\right) \approx \delta_{i} \frac{x_{i+1 / 2}-x_{i+1}}{x_{i+1}-x_{i}}<0,
$$

and

$$
\operatorname{sgn}\left(C_{0}\left(\gamma_{i+2}-\gamma_{i+1}\right)\right)=\operatorname{sgn}\left(\gamma_{i+1}-\gamma_{i+2}\right)=\operatorname{sgn}\left(\gamma_{i+1}\right) .
$$

This completes the proof of the sign-property for the second degree reconstruction with infinitely smooth RBFs of first order for small enough grids.

The proof for $N=3$ can be found in Appendix A.

\section{Entropy stable RBF-BASED MEthods}

In one space dimension there is no need to deviate from the polynomial reconstruction. For unstructured grids in multiple dimensions the problem is the construction of an interpolation function. There exist a lot cell or point configurations such that the reconstruction problem is not well-defined. This issue can be relaxed by solving an overdetermined system of equations, but then we lose the exact interpolation property. The RBFinterpolation can circumvent this problem since we do not need a unisolvent set of cells or points, but only a unisolvent subset of lower order. Thus, by adding some extra cells we significantly reduce the possibility that an unsolvable configuration occurs.

\subsection{RBF-TeCNOp method}

Based on the theory of entropy stable schemes and the work of Fjordholm et al. [11] we introduce the RBFTeCNOp scheme. By using Algorithm 1 with (4.21) for calculating the least oscillatory stencil, Theorem 5.9 shows that the sign-property holds for 2 nd and 3rd degree reconstruction in the limit of $\Delta x \rightarrow 0$. We conjecture that this result generalizes to higher order reconstructions. Thus, by combining the framework proposed in [11] with the RBF reconstruction using multiquadratics we recover an entropy stable essentially nonoscillatory RBF-based finite difference method of arbitrary high order. Furthermore, we use the RBF-RA algorithm to circumvent ill-conditioning in the reconstruction step [42].

In more detail, for constructing a $p$ th order RBF-TeCNOp method of the form (2.15) we use an entropy conservative flux of order $2 k$ with $k=\lceil p / 2\rceil$ (see Thm. 2.2) and an ENO based RBF reconstruction (Algorithm 1) on the scaled entropy variables of order $p$ with multiquadratics of order $p-1$.

Based on the Roe diffusion operator

$$
R|\Lambda| R^{-1} \llbracket u \rrbracket,
$$

with the eigenvector matrix $R$ and the diagonal matrix of the eigenvalues $\Lambda$, evaluated at the Roe average, we are choosing $R$ and $\Lambda$ in the same way. By Merriam [26] there is a scaling of the eigenvectors such that $R R^{T}=v_{u}=\partial_{u} v\left(u_{i+1 / 2}\right)$. Thus, we get the relation

$$
R|\Lambda| R^{-1} \llbracket u \rrbracket \approx R|\Lambda| R^{-1} v_{u} \llbracket v \rrbracket=R|\Lambda| R^{T} \llbracket v \rrbracket,
$$


that has a similar structure to that of a diffusion operator (2.16). The numerical diffusion term can be written as

$$
D_{i+1 / 2}\left\langle\langle v\rangle_{i+1 / 2}=R_{i+1 / 2} \Lambda_{i+1 / 2}\left\langle\langle w\rangle_{i+1 / 2},\right.\right.
$$

with the scaled entropy variables (2.18). Furthermore, we choose $\Lambda_{i+1 / 2}=\operatorname{diag}\left(\lambda_{1}\left(u_{i+1 / 2}\right), \ldots, \lambda_{N}\left(u_{i+1 / 2}\right)\right)$ and $u_{i+1 / 2}=\frac{u_{i}+u_{i+1}}{2}$ with the eigenvalues $\Lambda(u)$ of the Jacobian $\nabla_{u} f$.

It is important to note that the ill-conditioning of the interpolation matrix does not only affect the evaluation of the reconstruction; it also affects the calculation of the smoothness indicator which is based on the sum of the squares of the coefficients of the RBF-part of the interpolation.

From the theory we expect that the error of the interpolation with infinitely smooth RBFs decreases for smaller shape parameters. However, computations suggest that the choice of the stencil does not depend on the shape parameter. Thus, we calculate the stencil with respect to a stable shape parameter.

\subsection{RBF-finite volume method}

The combination of the RBF interpolation with finite volume methods works analogeously to the RBFTeCNOp Method. Aboiyar et al. [1] combine in their work a high-order WENO approach with a polyharmonic spline reconstruction and Bigoni et al. [3] apply a high-order WENO approach to multiquadratics.

We construct an entropy stable finite volume method of second order that is essentially nonoscillatory by combining (2.22) with a second order accurate RBF interpolation that acts on the scaled entropy variables. Therefore, we are using multiquadratics with the smoothness indicator (4.21), combined with Algorithm 1 and the vector valued rational approximation to ensure a stable evaluation of the interpolation function.

We conjecture the sign-property for the RBF reconstruction on mean values that is based on Algorithm 1 which is fulfilled in the pointwise case for second and third degree reconstruction in the limit $\Delta x \rightarrow 0$ (Thm. 5.9). Under this assumption we recover a second order entropy stable finite volume (RBF-EFV2) method. This method can be generalized to general grids in higher dimensions [32].

\section{Numerical RESUlts}

In this chapter, we are evaluating the second order entropy stable finite volume (EFV2) and the TeCNOp methods with RBF reconstruction for one-dimensional problems and compare it with its original version. Note that in one dimension we do not expect to do better than the classical methods, but also not worse.

For the polynomial reconstruction we use the original algorithm from [18] to select the stencil and in the RBF case we use Algorithm 1. The EFV2 and TeCNOp methods are based on the diffusion term (6.3).

The parameters for the vector-valued rational approximation are chosen as described in [42]. Further, we choose the shape parameter $\varepsilon=0.1$ for all examples.

\subsection{Linear advection equation}

We consider the linear advection equation

$$
u_{t}+a u_{x}=0
$$

with wave speed $a=1$ and periodic boundary conditions [25]. With the entropy function $\eta(u)=\frac{u^{2}}{2}$ we have

$$
q(u)=a \frac{u^{2}}{2}, \quad v(u)=u, \quad \psi(u)=a \frac{u^{2}}{2},
$$

and obtain the second order entropy conservative flux

$$
\tilde{F}_{i+1 / 2}=\bar{u}_{i+1 / 2},
$$


TABLE 2. Convergence rates of TeCNOp and EFV2 methods using multiquadratics and polynomials for the linear advection and Burgers equation on $[0,1]$ at time $t=0.1$.

\begin{tabular}{|c|c|c|c|c|c|c|c|c|c|}
\hline \multirow{3}{*}{$N$} & & \multicolumn{4}{|c|}{ Linear Advection equation } & \multicolumn{4}{|c|}{ Burgers' equation } \\
\hline & & \multicolumn{2}{|c|}{ RBF Reconstr } & \multicolumn{2}{|c|}{ Poly Reconstr } & \multicolumn{2}{|c|}{ RBF Reconstr } & \multicolumn{2}{|c|}{ Poly Reconstr } \\
\hline & & error & rate & error & rate & error & rate & error & rate \\
\hline \multirow[t]{5}{*}{$\mathrm{TeCNO} 2$} & 16 & $2.27 \mathrm{e}-02$ & - & $2.26 \mathrm{e}-02$ & - & $3.01 \mathrm{e}-02$ & - & $2.97 \mathrm{e}-02$ & - \\
\hline & 32 & $7.26 \mathrm{e}-03$ & 1.64 & $7.26 \mathrm{e}-03$ & 1.64 & $9.01 \mathrm{e}-03$ & 1.74 & $9.01 \mathrm{e}-03$ & 1.72 \\
\hline & 64 & $2.06 \mathrm{e}-03$ & 1.82 & $2.06 \mathrm{e}-03$ & 1.82 & $2.46 \mathrm{e}-03$ & 1.87 & $2.46 \mathrm{e}-03$ & 1.87 \\
\hline & 128 & $5.44 \mathrm{e}-04$ & 1.92 & $5.44 \mathrm{e}-04$ & 1.92 & $6.88 \mathrm{e}-04$ & 1.84 & $6.88 \mathrm{e}-04$ & 1.84 \\
\hline & 256 & $1.45 \mathrm{e}-04$ & 1.91 & $1.45 \mathrm{e}-04$ & 1.91 & $1.88 \mathrm{e}-04$ & 1.87 & $1.88 \mathrm{e}-04$ & 1.87 \\
\hline \multirow[t]{5}{*}{ TeCNO3 } & 16 & $1.48 \mathrm{e}-03$ & - & $1.48 \mathrm{e}-03$ & - & $5.19 \mathrm{e}-03$ & - & $5.17 \mathrm{e}-03$ & - \\
\hline & 32 & $1.89 \mathrm{e}-04$ & 2.97 & $1.89 \mathrm{e}-04$ & 2.97 & $9.23 \mathrm{e}-04$ & 2.49 & $9.23 \mathrm{e}-04$ & 2.49 \\
\hline & 64 & $2.36 \mathrm{e}-05$ & 3.00 & $2.36 \mathrm{e}-05$ & 3.00 & $1.47 \mathrm{e}-04$ & 2.65 & $1.47 \mathrm{e}-04$ & 2.65 \\
\hline & 128 & $2.96 \mathrm{e}-06$ & 3.00 & $2.96 \mathrm{e}-06$ & 3.00 & $2.52 \mathrm{e}-05$ & 2.54 & $2.52 \mathrm{e}-05$ & 2.54 \\
\hline & 256 & $3.70 \mathrm{e}-07$ & 3.00 & $3.70 \mathrm{e}-07$ & 3.00 & $4.13 \mathrm{e}-06$ & 2.61 & $4.13 \mathrm{e}-06$ & 2.61 \\
\hline \multirow[t]{5}{*}{$\overline{\mathrm{TeCNO} 4}$} & 16 & $5.61 \mathrm{e}-04$ & - & $5.60 \mathrm{e}-04$ & - & $2.84 \mathrm{e}-03$ & - & $2.84 \mathrm{e}-03$ & - \\
\hline & 32 & $3.98 \mathrm{e}-05$ & 3.82 & $3.98 \mathrm{e}-05$ & 3.82 & $5.37 \mathrm{e}-04$ & 2.40 & $5.37 \mathrm{e}-04$ & 2.40 \\
\hline & 64 & $2.62 \mathrm{e}-06$ & 3.92 & $2.62 \mathrm{e}-06$ & 3.93 & $5.76 \mathrm{e}-05$ & 3.22 & $5.76 \mathrm{e}-05$ & 3.22 \\
\hline & 128 & $1.74 \mathrm{e}-07$ & 3.91 & $1.74 \mathrm{e}-07$ & 3.90 & $4.97 \mathrm{e}-06$ & 3.54 & $4.97 \mathrm{e}-06$ & 3.54 \\
\hline & 256 & $1.14 \mathrm{e}-08$ & 3.93 & $1.14 \mathrm{e}-08$ & 3.93 & $6.97 \mathrm{e}-07$ & 2.83 & $6.97 \mathrm{e}-07$ & 2.83 \\
\hline \multirow[t]{5}{*}{ TeCNO5 } & 16 & $4.40 \mathrm{e}-05$ & - & $4.40 \mathrm{e}-05$ & - & $1.17 \mathrm{e}-03$ & - & $1.17 \mathrm{e}-03$ & - \\
\hline & 32 & $1.40 \mathrm{e}-06$ & 4.98 & $1.40 \mathrm{e}-06$ & 4.98 & $2.90 \mathrm{e}-04$ & 2.01 & $2.90 \mathrm{e}-04$ & 2.01 \\
\hline & 64 & $4.43 \mathrm{e}-08$ & 4.98 & $4.43 \mathrm{e}-08$ & 4.98 & $1.19 \mathrm{e}-05$ & 4.61 & $1.19 \mathrm{e}-05$ & 4.61 \\
\hline & 128 & $1.47 \mathrm{e}-09$ & 4.92 & $1.47 \mathrm{e}-09$ & 4.92 & $6.84 \mathrm{e}-07$ & 4.12 & $6.84 \mathrm{e}-07$ & 4.12 \\
\hline & 256 & $5.50 \mathrm{e}-11$ & 4.74 & $5.50 \mathrm{e}-11$ & 4.74 & $1.81 \mathrm{e}-07$ & 1.92 & $1.81 \mathrm{e}-07$ & 1.92 \\
\hline \multirow[t]{5}{*}{$\overline{\text { EFVM2 }}$} & 16 & $2.25 \mathrm{e}-02$ & - & $2.24 \mathrm{e}-02$ & - & $2.68 \mathrm{e}-02$ & - & $2.68 \mathrm{e}-02$ & - \\
\hline & 32 & $7.26 \mathrm{e}-03$ & 1.63 & $7.25 \mathrm{e}-03$ & 1.63 & $8.10 \mathrm{e}-03$ & 1.73 & $8.10 \mathrm{e}-03$ & 1.73 \\
\hline & 64 & $2.06 \mathrm{e}-03$ & 1.82 & $2.06 \mathrm{e}-03$ & 1.82 & $2.28 \mathrm{e}-03$ & 1.83 & $2.28 \mathrm{e}-03$ & 1.83 \\
\hline & 128 & $5.44 \mathrm{e}-04$ & 1.92 & $5.44 \mathrm{e}-04$ & 1.92 & $6.40 \mathrm{e}-04$ & 1.84 & $6.40 \mathrm{e}-04$ & 1.83 \\
\hline & 256 & $1.45 \mathrm{e}-04$ & 1.91 & $1.45 \mathrm{e}-04$ & 1.91 & $1.78 \mathrm{e}-04$ & 1.85 & $1.78 \mathrm{e}-04$ & 1.85 \\
\hline
\end{tabular}

Notes. We use periodic boundary conditions and $u_{0}(x)=\sin (2 \pi x)$, shape parameter $\varepsilon=0.1, \mathrm{CFL}=0.5$.

TABLE 3. Runtime comparison for the 5th order method solving the linear advection equation.

\begin{tabular}{llll}
\hline & RBF + RBF-RA & RBF & Poly \\
\hline 16 & 4.3 & 3.2 & 2.1 \\
32 & 7.4 & 5.0 & 3.7 \\
64 & 21.8 & 12.6 & 6.6 \\
128 & 73.5 & 38.0 & 16.9 \\
256 & 279.5 & 132.4 & 44.9 \\
\hline
\end{tabular}

to construct a high-order accurate scheme. We use a 5th order SSPRK method for the time discretization in the TeCNOp method [17]. For the EFV2 method we use the second order entropy conservative flux plus a third order SSPRK method in time.

The convergence results for the smooth initial conditions are shown in Table 2 . The $L_{1}$-errors are the same for the different reconstruction methods for grids of size smaller than 1/32 and their convergence rates are as expected.

In Table 3 we present a comparison of the runtime for the 5 th order TeCNO scheme with RBF reconstruction using the RBF-RA algorithm, RBF reconstruction evaluated at a stable shape parameter. The RBF method 

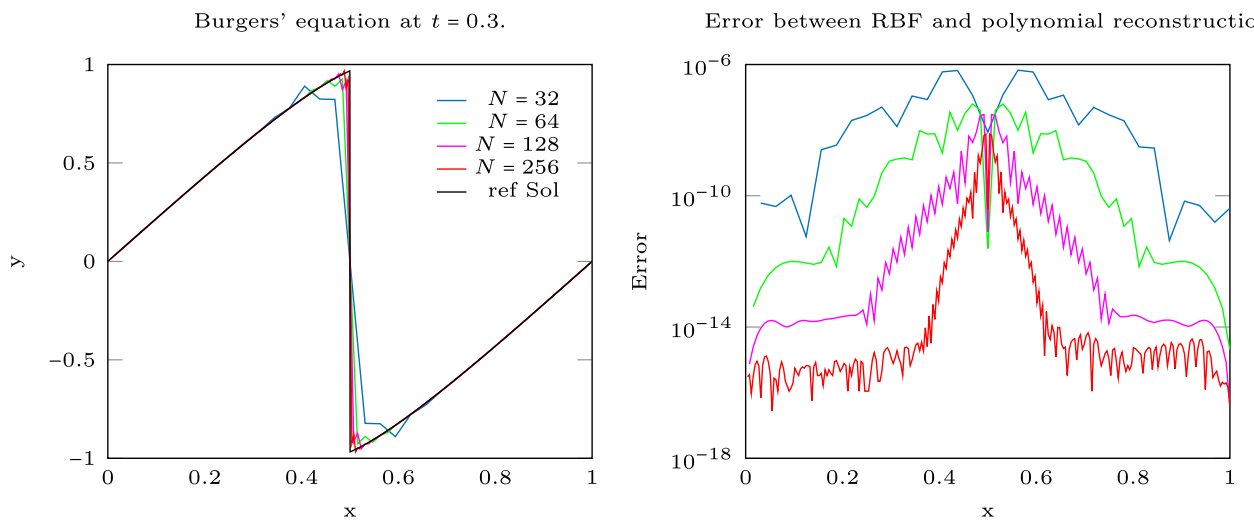

Figure 1. Burgers' equation on $[0,1]$ at time $t=0.3$ with continuous initial condition $u_{0}=$ $\sin (2 \pi x)$, shape parameter $\varepsilon=0.1, \mathrm{CFL}=0.5$, solved by TeCNO5.

based on the RBF-RA algorithm solves multiple times the same system of equations with different shape parameters to approximate the final one, explaining the difference between the first two columns. The difference between the RBF and the polynomial reconstruction comes from the fact that the RBF algorithm solves in each recursive step of Algorithm 1 a system of equations and the polynomial case calculates just the next divided difference.

\subsection{Burgers' equation}

For the Burgers equation

$$
u_{t}+\frac{1}{2}\left(u^{2}\right)_{x}=0,
$$

we study the order of convergence and check if the methods handle discontinuities without introducing major oscillations. The EFV2 and TeCNOp method are based on the entropy $\eta(u)=u^{2} / 2$ and

$$
q(u)=\frac{u^{3}}{3}, \quad v(u)=u, \quad \psi(u)=\frac{u^{3}}{6},
$$

leading to an entropy conservative flux

$$
\tilde{F}_{i+1 / 2}=\frac{u_{i}^{2}+u_{i} u_{i+1}+u_{i+1}^{2}}{6}
$$

which is used to construct an high-order scheme. For the time discretization we use a 5th order SSPRK method [17]. Furthermore, we choose the domain [0,1] and the initial conditions $u_{0}(x)=\sin (2 \pi x)$.

A detailed analysis of the convergence is shown in Table 2. The convergence rate is as expected and the errors of the two different methods (polynomial reconstruction and RBF reconstruction) coincide. At time $t=0.3$ a discontinuity emerges at $x=0.5$. This can be resolved accurately with vanishing oscillations (Fig. 1). Furthermore, we observe that the difference between the reconstruction methods vanishes in the smooth part while at the shock it stays small.

\subsection{Shallow water equations}

For the shallow water equations (2.6) we consider the dambreak problem with the initial conditions

$$
\left(h_{0}, m_{0}\right)=\left\{\begin{array}{ll}
(1.5,0) & \text { if }|x| \leqslant 0.2 \\
(1,0) & \text { if }|x|>0.2
\end{array},\right.
$$



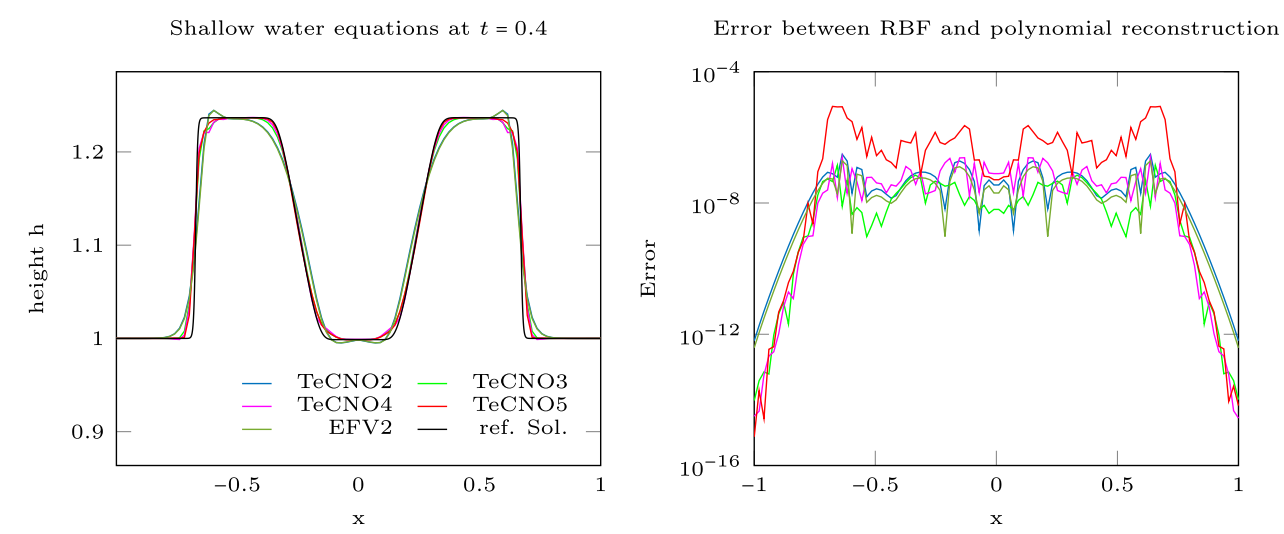

Figure 2. Shallow water equations on $[-1,1]$ at time $t=0.4$ with $N=100$, shape parameter $\varepsilon=0.1, \mathrm{CFL}=0.5$, solved by $\mathrm{TeCNO}$ and $\mathrm{EFV} 2$.

on the domain $[-1,1]$ and periodic boundary conditions. We use a second order entropy stable flux (2.9) to construct a high-order flux and a third order SSPRK method for the time integration.

Fjordholm et al. [11] showed that the standard TeCNO scheme behaves similar to the ENO-MUSCL scheme. The same holds for the RBF-TeCNOp scheme and the RBF-EFV2 scheme as seen in Figure 2. The difference between the RBF methods and the polynomial scheme is around $\mathcal{O}\left(10^{-6}\right)$ in the region where the discontinuity passed and much smaller in smooth regions.

\subsection{Euler equations}

The one-dimensional Euler equations (2.10) are a system of size three. As for the shallow water equations we use a third order SSPRK method and as a second order entropy conservative flux we use the KEPEC-flux (2.13). Further, we choose $\gamma=1.4$ which simulates a diatomic gas such as air.

\subsubsection{Sod's shock tube problem}

Sod's shock tube problem is a Riemann problem where two gases with different densities collide. A rarefaction wave emerges, followed by a contact and a shock discontinuity. The initial conditions are

$$
\left(\rho_{0}, m_{0}, p_{0}\right)=\left\{\begin{array}{ll}
(1,0,1) & \text { if } x<0 \\
(0.125,0,0.1) & \text { if } x \geqslant 0
\end{array},\right.
$$

where $m=u \rho$. The results at time $t=2$ of the RBF-TeCNOp and RBF-EFV2 methods are shown in Figure 3, clearly representing the rarefaction wave, the contact, and the shock discontinuity. Comparing the solutions obtained with polynomial reconstruction or with RBF reconstruction, we see in Figure 4 that their difference is decreasing with the refinement of the grid.

\subsubsection{Lax shock tube problem}

The Lax shock tube problem is another Riemann problem with the initial conditions

$$
\left(\rho_{0}, m_{0}, p_{0}\right)=\left\{\begin{array}{ll}
(0.445,0.698,3.528) & \text { if } x<0 \\
(0.5,0,0.571) & \text { if } x \geqslant 0
\end{array},\right.
$$

where $m=u \rho$. The RBF-TeCNOp methods of order three to five represent the big shock in the density sharply with just $N=100$ points, see Figure 5 . The second order RBF-EFV2 method does not perform well for this case. 

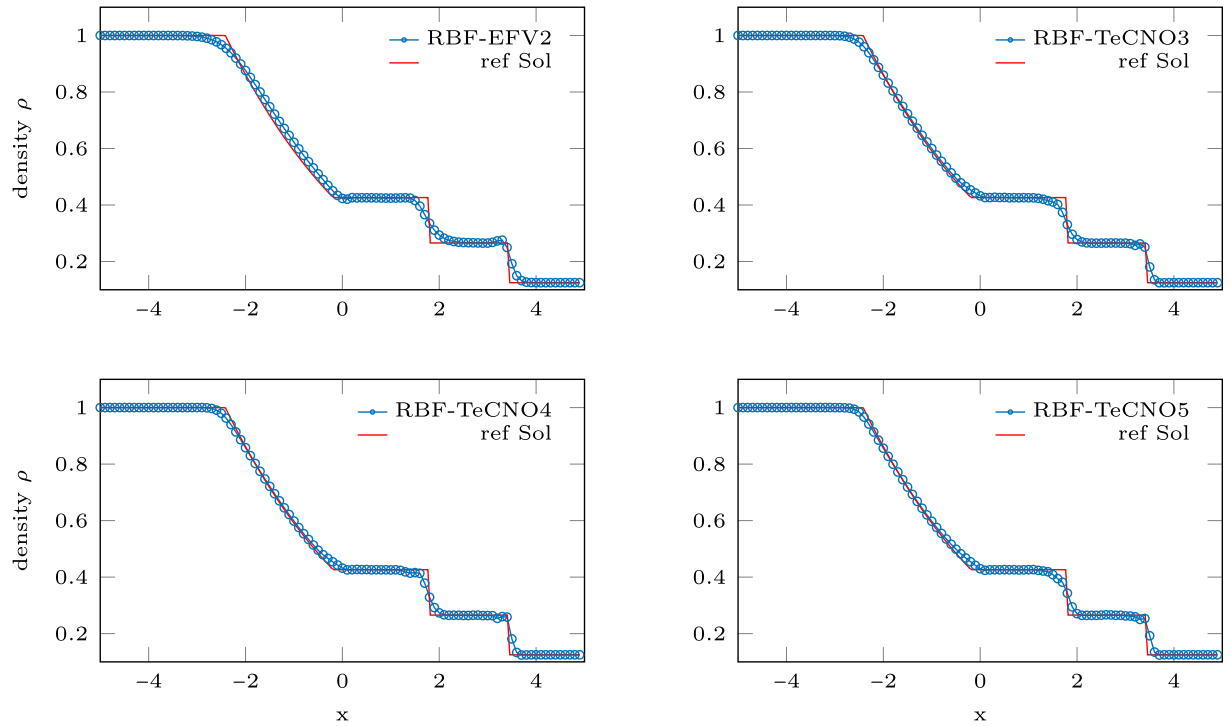

FiguRE 3. Sod's shock tube problem on $[-5,5]$ at time $t=2$ with $N=100$, shape parameter $\varepsilon=0.1, \mathrm{CFL}=0.3$, solved by RBF-TeCNOp and RBF-EFV2.

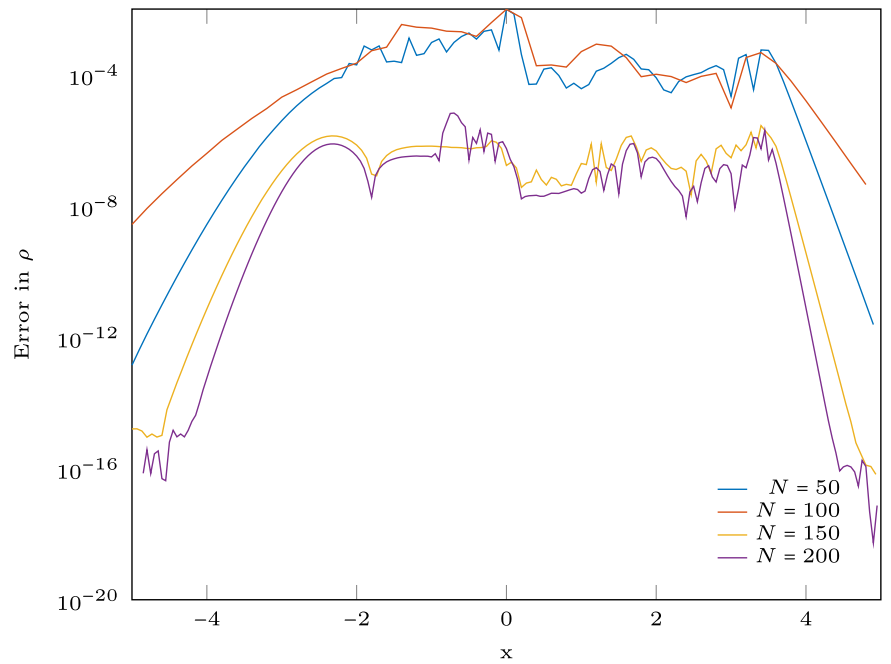

FIGURE 4. Pointwise error between RBF and polynomial based reconstruction EFV2 method for the Sod's shock tube problem on $[-5,5]$ at time $t=2$ for different number of grid points $N$, shape parameter $\varepsilon=0.1, \mathrm{CFL}=0.3$.

\subsubsection{Shu-Osher shock-entropy wave interaction problem}

The Shu-Osher problem models a shock-turbulence interaction in which a shock interacts with a low frequency wave. Due to this interaction high-frequency oscillations develop over time. The initial conditions are

$$
\left(\rho_{0}, m_{0}, p_{0}\right)=\left\{\begin{array}{ll}
(3.857143,2.629369,10.33333) & \text { if } x<-4 \\
(1+0.2 \sin (5 x), 0,1) & \text { if } x \geqslant-4
\end{array},\right.
$$



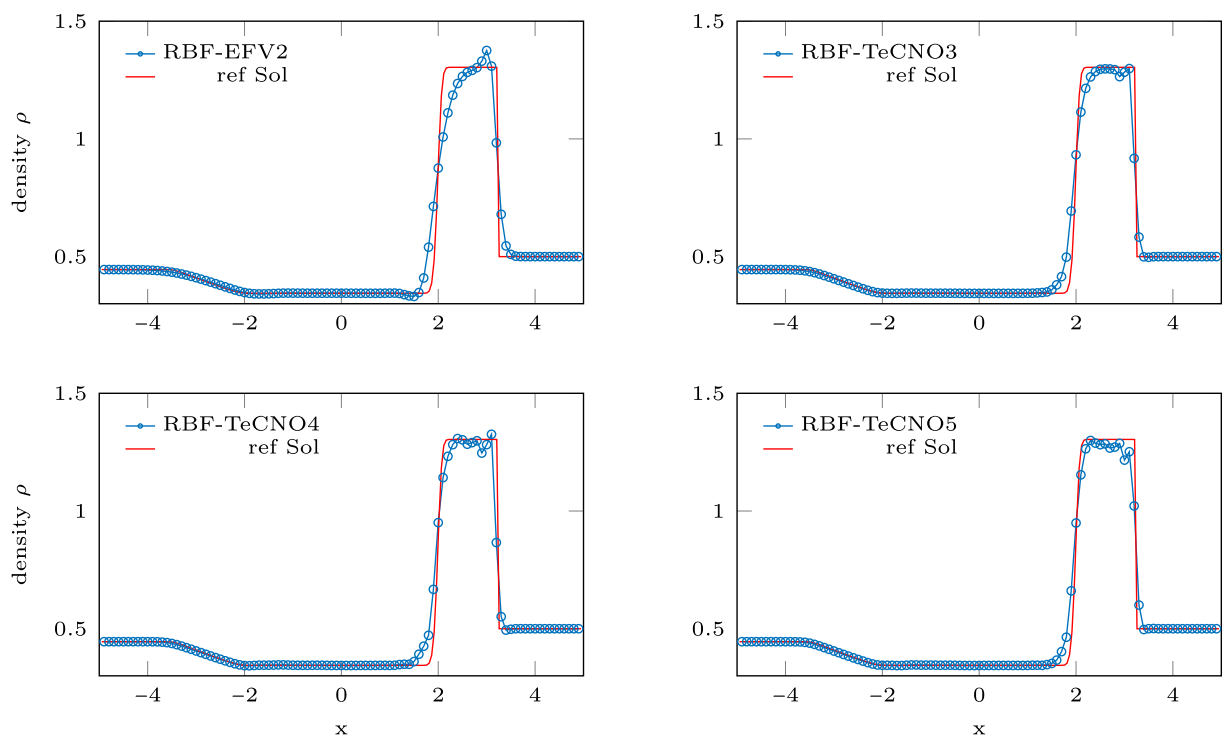

Figure 5. Lax shock tube problem on $[-5,5]$ at time $t=1.3$ with $N=100$, shape parameter $\varepsilon=0.1, \mathrm{CFL}=0.3$, solved by RBF-TeCNOp and RBF-EFV2.
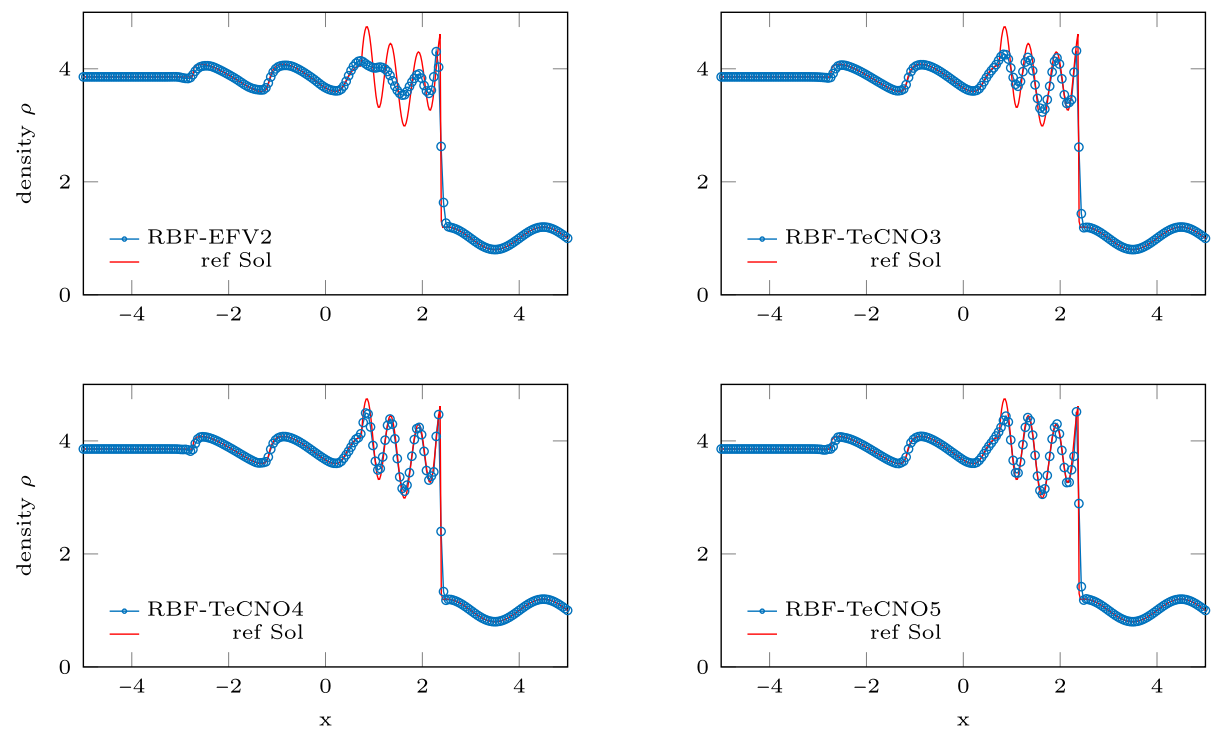

FiguRE 6 . Shu-Osher problem on $[-5,5]$ at time $t=1.8$ with $N=200$, shape parameter $\varepsilon=0.1, \mathrm{CFL}=0.3$ solved by RBF-TeCNOp and RBF-EFV2.

where $m=u \rho$. The RBF-TeCNOp methods of order larger than three recover the high frequency oscillations well. The RBF-EFV2 method fits the low order oscillations and the shock, but not the high frequency one due to excessive dissipation (Fig. 6). 

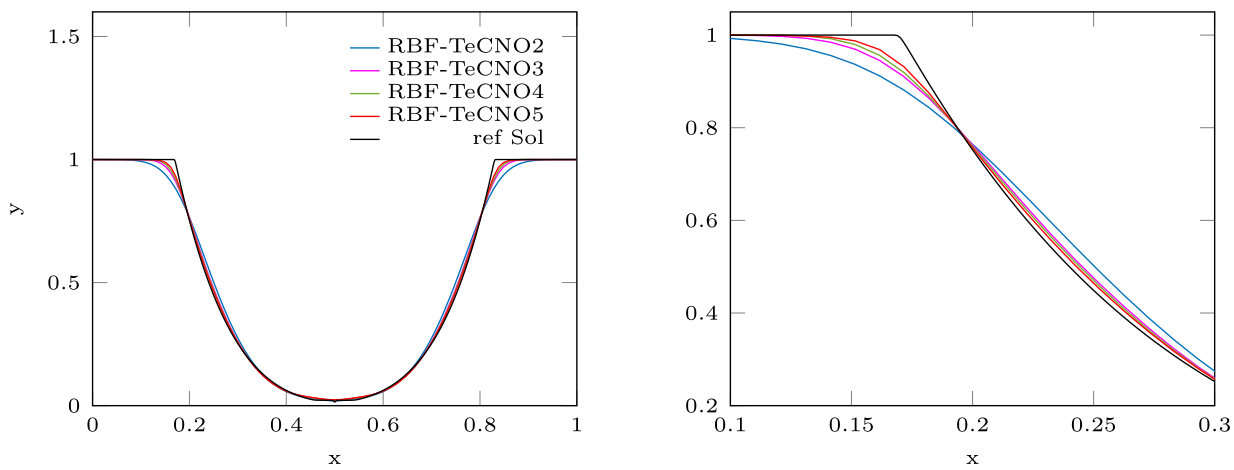

Figure 7 . Low density problem on $[0,1]$ at time $t=0.12$ with $N=100$, shape parameter $\varepsilon=0.1, \mathrm{CFL}=0.1$ solved by RBF-TeCNOp.
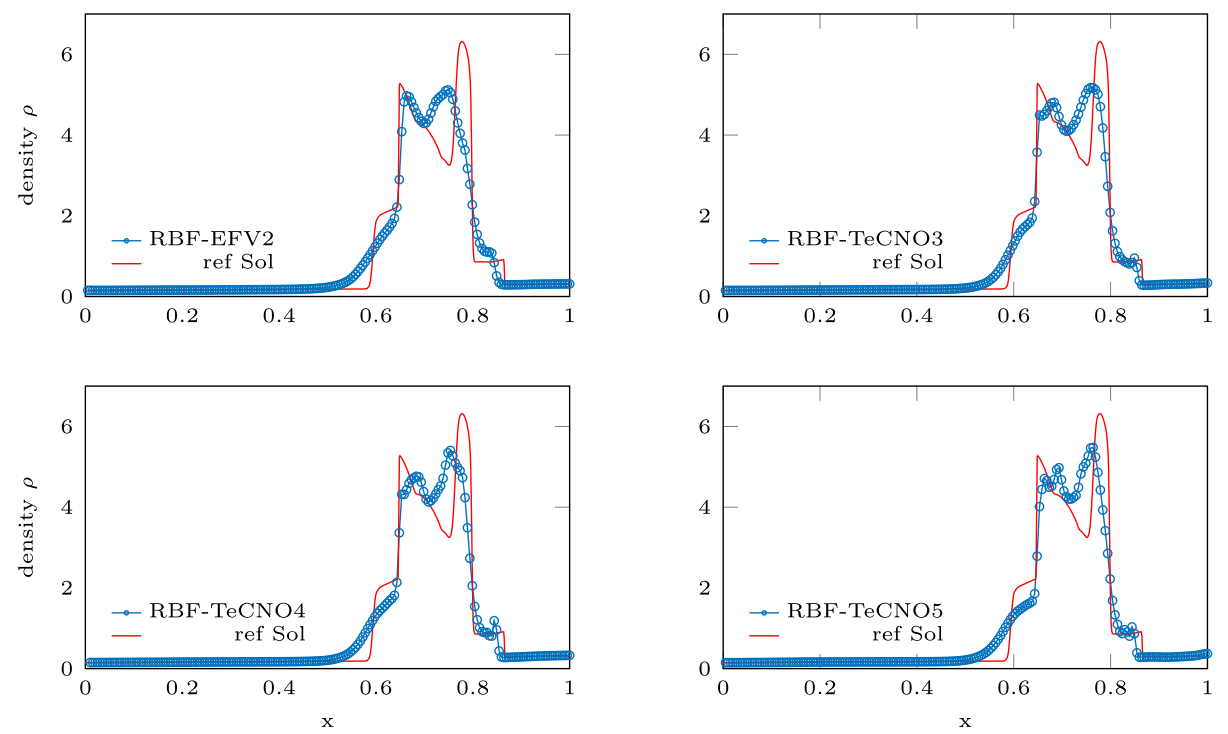

FiguRe 8 . WC blast wave problem on $[0,1]$ at time $t=0.038$ with $N=200$, shape parameter $\varepsilon=0.1, \mathrm{CFL}=0.1$ solved by RBF-TeCNOp and RBF-EFV2.

\subsubsection{Low density problem}

The low density problem on $[0,1]$ is a Riemann problem that tests the ability of preserving positive density and pressure. The initial condition are

$$
\left(\rho_{0}, m_{0}, p_{0}\right)= \begin{cases}(1,-2,0.4) & \text { if } x<0.5 \\ (1,2,0.4) & \text { if } x \geqslant 0.5\end{cases}
$$

with Neumann boundary conditions.

In Figure 7 we observe the increasing accuracy of the RBF-TeCNOp method with increasing order $p$. Note that by choosing the wrong smoothness indicator, negative pressures will occur. 


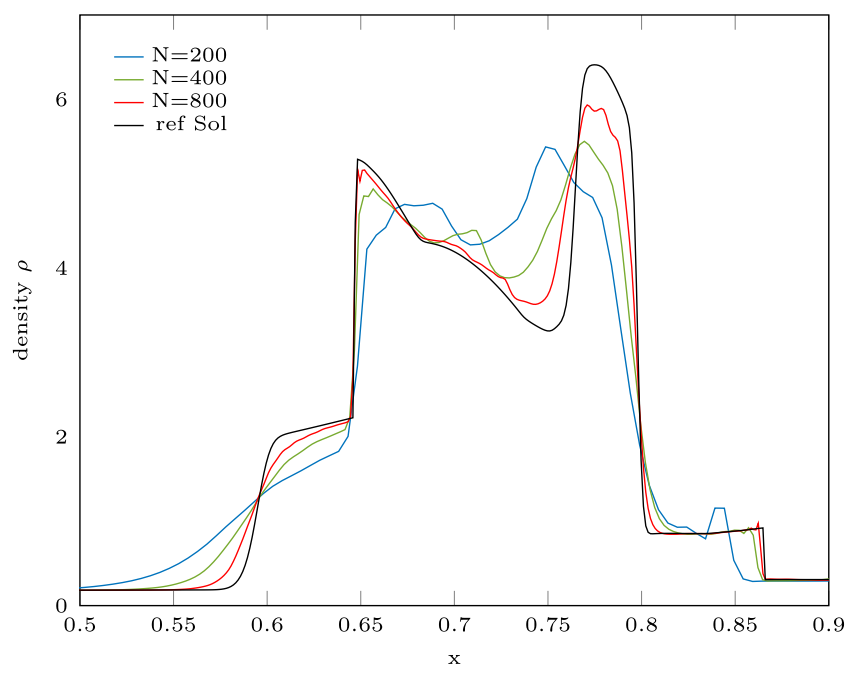

Figure 9. WC blast wave problem on $[0,1]$ at time $t=0.038$ with shape parameter $\varepsilon=0.1$, $\mathrm{CFL}=0.1$ solved by RBF-TeCNO4.

\subsubsection{Two interacting blast waves}

A more complex one dimensional example is the two interacting blast waves, introduced by Woodward et al. [41]. It is based on two blast waves that interact and introduce low pressures and densities. Its initial condition is

$$
\left(\rho_{0}, m_{0}, p_{0}\right)= \begin{cases}(1,0,1000) & \text { if } x<0.1 \\ (1,0,0.01) & \text { if } 0.1 \leqslant x<0.9 \\ (1,0,100) & \text { if } x \geqslant 0.9\end{cases}
$$

Compared to the low density problem we add here an additional challenge caused by the collision of the two shocks. Note that the standard version of the TeCNO method always produces negative pressures or densities for the RBF and polynomial reconstruction.

Thus, we introduce a more complicated symmetric positive definite dissipation operator (2.16), similar to the one introduced by Derigs et al. [6]. The goal is to mimic the more dissipative Rusanov-type diffusion operator such that

$$
\alpha \llbracket u \rrbracket_{i+1 / 2}=D_{i+1 / 2} \llbracket v \rrbracket_{i+1 / 2} .
$$

See Appendix B for more details.

The results with the new dissipation matrix approximate the correct solution and we can see an improvement with increasing order (Fig. 8) and for increasing number of points (Fig. 9).

\section{Conclusions}

We introduce a new smoothness indicator and an algorithm to choose the least oscillatory stencil based on $\mathrm{RBF}$ interpolation. This smoothness indicator is directly related to the RBF interpolation and it is based on the generalized divided difference method. For this ENO reconstruction we prove the sign-property in the finite difference case for the second and third order reconstruction in the limit $\Delta x \rightarrow 0$ for infinitely smooth RBFs on general grids. Further, we conjecture this property for higher order schemes and for the case of the average-based interpolation based on numerical experiments. Note that the condition $\Delta x \rightarrow 0$ can be replaced by the condition for the shape parameter that $\varepsilon \rightarrow 0$. 
Based on this, we construct a RBF-TeCNOp method as an arbitrary high-order entropy stable finite difference method and the RBF-EFV2 method as a second order entropy stable finite volume method. Both are based on high-order entropy conservative schemes and a diffusion term which depends on the RBF-reconstruction in the scaled entropy variables. To circumvent the ill-conditioning of the local interpolation problems we apply the vector-valued rational approximation method [42].

Thus, we propose a method that has all the properties of the original TeCNO scheme [11]. It is entropy stable, high-order accurate for smooth solutions and essentially nonoscillatory near discontinuities. To show their robustness we present a range of numerical simulations in one dimension. The solutions coincide up to a small error with those obtained from the original TeCNO method.

The main drawback of the method is the expensive evaluation of the vector-valued approximation to circumvent ill-conditioning, but this problem is being considered. The advantages of RBF-based reconstructions will become much clearer for high-dimensional problems and we hope to report on this in the near future.

\section{Appendix A. Proof of Theorem 5.9 For the 3Rd Degree reconstruction}

Proof. The proof is based on a study of all possible choices of stencils, that may result from Algorithm 1:

- $S_{i}=\left\{C_{i-2}, C_{i-1}, C_{i}\right\}, S_{i+1}=\left\{C_{i-1}, C_{i}, C_{i+1}\right\}$,

- $S_{i}=\left\{C_{i-2}, C_{i-1}, C_{i}\right\}, S_{i+1}=\left\{C_{i}, C_{i+1}, C_{i+2}\right\}$,

- $S_{i}=\left\{C_{i-2}, C_{i-1}, C_{i}\right\}, S_{i+1}=\left\{C_{i+1}, C_{i+2}, C_{i+3}\right\}$,

$-\ldots$

We consider $N=3$ (third degree reconstruction) and assume $\phi$ is of second order. The main difference between the second and third degree is that Algorithm 1 now gives two conditions for each stencil that depend on different grid sizes. Therefore, we introduce the superscript $l$ to indicate the size of the stencil

$$
\delta_{k}^{l}=\mathrm{d} \varphi^{k}\left(x_{k}\right)^{1 / 2}, \quad \beta_{k}^{l}=y_{k}-\sum_{i=1}^{l-1} y_{k-l} L_{k-l}^{k, l}\left(x_{l}\right), \quad \gamma_{k}^{l}=\frac{\beta_{k}^{l}}{\delta_{k}^{l}},
$$

based on the stencil $\left\{C_{k-l+1}, \cdots, C_{k}\right\}$. Further, we can show with simple calculations that

$$
\beta_{k+1}^{3}=\beta_{k+1}^{2}-\frac{x_{k+1}-x_{k}}{x_{k}-x_{k-1}} \beta_{k}^{2} .
$$

From (5.32) and (5.44) we recover

$$
\frac{x_{k+1}-x_{k}}{x_{k}-x_{k-1}} \approx \frac{\delta_{k+1}^{2}}{\delta_{k}^{2}}
$$

which allows us to conclude that

$$
\delta_{k+1}^{3} \gamma_{k+1}^{3}=\beta_{k+1}^{3} \approx \beta_{k+1}^{2}-\frac{\delta_{k+1}^{2}}{\delta_{k}^{2}} \beta_{k}^{2}=\delta_{k+1}^{2}\left(\gamma_{k+1}^{2}-\gamma_{k}^{2}\right),
$$

and so

$$
\gamma_{k+1}^{3}=\frac{\delta_{k+1}^{2}}{\delta_{k+1}^{3}}\left(\gamma_{k+1}^{2}-\gamma_{k}^{2}\right)
$$

Note that the term $\delta_{k+1}^{2} / \delta_{k+1}^{3}$ is always positive (Cor. 4.4). Next, we can show the sign of the constant $C_{l}$ using Theorem 5.1

$$
\begin{aligned}
C_{0} & =\delta_{k}\left(\frac{\mathrm{d} \varphi^{k}\left(x_{i+1 / 2}\right)}{\mathrm{d} \varphi^{k}\left(x_{k}\right)}-A_{k}\right) \approx \delta_{k}\left(\frac{\left(x_{k-2}-x_{i+1 / 2}\right)\left(x_{k-1}-x_{i+1 / 2}\right)}{\left(x_{k-2}-x_{k}\right)\left(x_{k-1}-x_{k}\right)}-\frac{x_{i+1 / 2}-x_{k-1}}{x_{k}-x_{k-1}}\right), \\
& \approx \delta_{k} \frac{\left(x_{i+1 / 2}-x_{k-1}\right)\left(x_{i+1 / 2}-x_{k}\right)}{\left(x_{k}-x_{k-2}\right)\left(x_{k}-x_{k-1}\right)}
\end{aligned}
$$


with $k=i+r_{N-1}+N-1$. By induction one proves that

$$
C_{l} \approx \delta_{k} \frac{x_{i+1 / 2}-x_{k+l-1}}{x_{k}-x_{k-2}} \frac{x_{i+1 / 2}-x_{k+l}}{x_{k}-x_{k-1}},
$$

for $l \in \mathbb{N}$ and we recover

$$
\operatorname{sgn}\left(C_{l}\right)=(-1)^{r_{N-1}+N-1+l}
$$

Case 1. Consider the stencils $S_{i}=\left\{C_{i-2}, C_{i-1}, C_{i}\right\}, S_{i+1}=\left\{C_{i-1}, C_{i}, C_{i+1}\right\}$, equivalent to the conditions

$$
\left|\gamma_{i}^{2}\right|<\left|\gamma_{i+1}^{2}\right|, \quad\left|\gamma_{i}^{3}\right|<\left|\gamma_{i+1}^{3}\right|, \quad\left|\gamma_{i+1}^{2}\right|<\left|\gamma_{i+2}^{2}\right|, \quad\left|\gamma_{i+1}^{3}\right|<\left|\gamma_{i+2}^{3}\right|
$$

Note that this case can be characterized by $d_{2}=1$ and $s_{2}=r_{2}=-2$. From Theorem 5.8 we know

$$
j R_{i+1 / 2} \approx C_{0}\left(\gamma_{i+1}^{3}-\gamma_{i}^{3}\right),
$$

and we recover

$$
\operatorname{sgn}\left(C_{0}\left(\gamma_{i+1}^{3}-\gamma_{i}^{3}\right)\right)=\operatorname{sgn}\left(\gamma_{i+1}^{3}-\gamma_{i}^{3}\right)=\operatorname{sgn}\left(\gamma_{i+1}^{3}\right)=\operatorname{sgn}\left(\gamma_{i+1}^{2}-\gamma_{i}^{2}\right)=\operatorname{sgn}\left(\gamma_{i+1}^{2}\right)=\operatorname{sgn}\left(y_{i+1}-y_{i}\right),
$$

where we used (A.1), (A.2) and (A.4).

Case 2. Assume the stencil $S_{i}=\left\{C_{i-2}, C_{i-1}, C_{i}\right\}, S_{i+1}=\left\{C_{i}, C_{i+1}, C_{i+2}\right\}$, equivalent to the conditions

$$
\begin{aligned}
& \left|\gamma_{i}^{2}\right|<\left|\gamma_{i+1}^{2}\right|, \quad\left|\gamma_{i}^{3}\right|<\left|\gamma_{i+1}^{3}\right|, \\
& \left\{\begin{array}{lll}
\left|\gamma_{i+1}^{2}\right|<\left|\gamma_{i+2}^{2}\right|, & \left|\gamma_{i+1}^{3}\right|>\left|\gamma_{i+2}^{3}\right|, \quad(a) \\
\left|\gamma_{i+1}^{2}\right|>\left|\gamma_{i+2}^{2}\right|, & \left|\gamma_{i+2}^{3}\right|<\left|\gamma_{i+3}^{3}\right| . & (b)
\end{array}\right.
\end{aligned}
$$

The jump can be written by

$$
j R_{i+1 / 2} \approx C_{0}\left(\gamma_{i+1}^{3}-\gamma_{i}^{3}\right)+C_{1}\left(\gamma_{i+2}^{3}-\gamma_{i+1}^{3}\right) .
$$

For each term we calculate its sign. The first term can be understood in the same way as above and it holds for both $(a)$ and $(b)$ in (A.6)

$$
\operatorname{sgn}\left(C_{0}\left(\gamma_{i+1}^{3}-\gamma_{i}^{3}\right)\right)=\operatorname{sgn}\left(y_{i+1}-y_{i}\right)
$$

For the second term we first assume that $(a)$ holds and compute its sign as

$$
\operatorname{sgn}\left(C_{1}\left(\gamma_{i+2}^{3}-\gamma_{i+1}^{3}\right)\right)=\operatorname{sgn}\left(\gamma_{i+1}^{3}-\gamma_{i+2}^{3}\right)=\operatorname{sgn}\left(\gamma_{i+1}^{3}\right)=\operatorname{sgn}\left(\gamma_{i+1}^{2}-\gamma_{i}^{2}\right)=\operatorname{sgn}\left(\gamma_{i+1}^{2}\right)=\operatorname{sgn}\left(y_{i+1}-y_{i}\right) .
$$

For $(b)$ we split it in two terms using (A.1)

$$
C_{1}\left(\gamma_{i+2}^{3}-\gamma_{i+1}^{3}\right)=C_{1} \frac{\delta_{i+2}^{2}}{\delta_{i+2}^{3}}\left(\gamma_{i+2}^{2}-\gamma_{i+1}^{2}\right)-C_{1} \frac{\delta_{i+1}^{2}}{\delta_{i+1}^{3}}\left(\gamma_{i+1}^{2}-\gamma_{i}^{2}\right),
$$

and calculate the sign of each one

$$
\begin{gathered}
\operatorname{sgn}\left(C_{1} \frac{\delta_{i+2}^{2}}{\delta_{i+2}^{3}}\left(\gamma_{i+2}^{2}-\gamma_{i+1}^{2}\right)\right)=\operatorname{sgn}\left(\gamma_{i+1}^{2}-\gamma_{i+2}^{2}\right)=\operatorname{sgn}\left(\gamma_{i+1}^{2}\right)=\operatorname{sgn}\left(y_{i+1}-y_{i}\right), \\
\left.\operatorname{sgn}\left(-C_{1} \frac{\delta_{i+1}^{2}}{\delta_{i+1}^{3}}\left(\gamma_{i+1}^{2}-\gamma_{i}^{2}\right)\right)=\operatorname{sgn}\left(\gamma_{i+1}^{2}-\gamma_{i}^{2}\right)\right)=\operatorname{sgn}\left(\gamma_{i+1}^{2}\right)=\operatorname{sgn}\left(y_{i+1}-y_{i}\right) .
\end{gathered}
$$


Case 3. Consider the stencil $S_{i}=\left\{C_{i-2}, C_{i-1}, C_{i}\right\}, S_{i+1}=\left\{C_{i+1}, C_{i+2}, C_{i+3}\right\}$, equivalent to the conditions

$$
\left|\gamma_{i}^{2}\right|<\left|\gamma_{i+1}^{2}\right|, \quad\left|\gamma_{i}^{3}\right|<\left|\gamma_{i+1}^{3}\right|, \quad\left|\gamma_{i+1}^{2}\right|>\left|\gamma_{i+2}^{2}\right|, \quad\left|\gamma_{i+2}^{3}\right|>\left|\gamma_{i+3}^{3}\right|
$$

For the reconstructed jump we have

$$
j R_{i+1 / 2} \approx C_{0}\left(\gamma_{i+1}^{3}-\gamma_{i}^{3}\right)+C_{1}\left(\gamma_{i+2}^{3}-\gamma_{i+1}^{3}\right)+C_{2}\left(\gamma_{i+3}^{3}-\gamma_{i+2}^{3}\right) .
$$

The sign of each term is

$$
\operatorname{sgn}\left(C_{0}\left(\gamma_{i+1}^{3}-\gamma_{i}^{3}\right)\right)=\operatorname{sgn}\left(\gamma_{i+1}^{3}-\gamma_{i}^{3}\right)=\operatorname{sgn}\left(\gamma_{i+1}^{3}\right)=\operatorname{sgn}\left(\gamma_{i+1}^{2}-\gamma_{i}^{2}\right)=\operatorname{sgn}\left(\gamma_{i+1}^{2}\right)=\operatorname{sgn}\left(y_{i+1}-y_{i}\right) .
$$

For the second term we need

$$
\begin{gathered}
\operatorname{sgn}\left(\gamma_{i+1}^{3}\right)=\operatorname{sgn}\left(\gamma_{i+1}^{2}-\gamma_{i}^{2}\right)=\operatorname{sgn}\left(\gamma_{i+1}^{2}\right), \\
\operatorname{sgn}\left(-\gamma_{i+2}^{3}\right)=\operatorname{sgn}\left(\gamma_{i+1}^{2}-\gamma_{i+2}^{2}\right)=\operatorname{sgn}\left(\gamma_{i+1}^{2}\right),
\end{gathered}
$$

such that we can show

$$
\operatorname{sgn}\left(C_{1}\left(\gamma_{i+2}^{3}-\gamma_{i+1}^{3}\right)\right)=\operatorname{sgn}\left(\gamma_{i+1}^{3}-\gamma_{i+2}^{3}\right)=\operatorname{sgn}\left(\gamma_{i+1}^{2}\right)=\operatorname{sgn}\left(y_{i+1}-y_{i}\right) .
$$

The last term yields

$$
\operatorname{sgn}\left(C_{2}\left(\gamma_{i+3}^{3}-\gamma_{i+2}^{3}\right)\right)=\operatorname{sgn}\left(\gamma_{i+3}^{3}-\gamma_{i+2}^{3}\right)=-\operatorname{sgn}\left(\gamma_{i+2}^{3}\right)=\operatorname{sgn}\left(\gamma_{i+1}^{2}-\gamma_{i+2}^{2}\right)=\operatorname{sgn}\left(\gamma_{i+1}^{2}\right)=\operatorname{sgn}\left(y_{i+1}-y_{i}\right) .
$$

Case 4. Assume the stencils $S_{i}=\left\{C_{i-1}, C_{i}, C_{i+1}\right\}, S_{i+1}=\left\{C_{i}, C_{i+1}, C_{i+2}\right\}$, equivalent to the conditions

$$
\begin{aligned}
& \left\{\begin{array}{l}
\left|\gamma_{i}^{2}\right|<\left|\gamma_{i+1}^{2}\right|, \quad\left|\gamma_{i}^{3}\right| \geqslant\left|\gamma_{i+1}^{3}\right|, \quad(a 1) \\
\left|\gamma_{i}^{2}\right| \geqslant\left|\gamma_{i+1}^{2}\right|, \quad\left|\gamma_{i+1}^{3}\right|<\left|\gamma_{i+2}^{3}\right|, \quad(a 2)
\end{array}\right. \\
& \begin{cases}\left|\gamma_{i+1}^{2}\right|<\left|\gamma_{i+2}^{2}\right|, \quad\left|\gamma_{i+1}^{3}\right| \geqslant\left|\gamma_{i+2}^{3}\right|, \quad(b 1) \\
\left|\gamma_{i+1}^{2}\right| \geqslant\left|\gamma_{i+2}^{2}\right|, \quad\left|\gamma_{i+2}^{3}\right|<\left|\gamma_{i+3}^{3}\right| . \quad(b 2)\end{cases}
\end{aligned}
$$

Here, we have the different combinations $\left(a_{1}, b_{1}\right),\left(a_{1}, b_{2}\right),\left(a_{2}, b_{1}\right)$ and $\left(a_{2}, b_{2}\right)$, where $\left(a_{2}, b_{1}\right)$ is not possible. The jump is represented as

$$
j R_{i+1 / 2} \approx C_{0}\left(\gamma_{i+2}^{3}-\gamma_{i+1}^{3}\right) .
$$

Let us first consider the combination $\left(a_{1}, b_{1}\right)$. We have

$$
\operatorname{sgn}\left(C_{0}\left(\gamma_{i+2}^{3}-\gamma_{i+1}^{3}\right)\right)=\operatorname{sgn}\left(\gamma_{i+1}^{3}-\gamma_{i+2}^{3}\right)=\operatorname{sgn}\left(\gamma_{i+1}^{3}\right)=\operatorname{sgn}\left(\gamma_{i+1}^{2}-\gamma_{i}^{2}\right)=\operatorname{sgn}\left(\gamma_{i+1}^{2}\right)=\operatorname{sgn}\left(y_{i+1}-y_{i}\right) .
$$

In the case $\left(a_{1}, b_{2}\right)$, we precalculate

$$
\begin{gathered}
\operatorname{sgn}\left(\gamma_{i+1}^{3}\right)=\operatorname{sgn}\left(\gamma_{i+1}^{2}-\gamma_{i}^{2}\right)=\operatorname{sgn}\left(\gamma_{i+1}^{2}\right), \\
\operatorname{sgn}\left(-\gamma_{i+2}^{3}\right)=\operatorname{sgn}\left(\gamma_{i+1}^{2}-\gamma_{i+2}^{2}\right)=\operatorname{sgn}\left(\gamma_{i+1}^{2}\right) .
\end{gathered}
$$

Thus,

$$
\operatorname{sgn}\left(C_{0}\left(\gamma_{i+2}^{3}-\gamma_{i+1}^{3}\right)\right)=\operatorname{sgn}\left(\gamma_{i+1}^{3}-\gamma_{i+2}^{3}\right)=\operatorname{sgn}\left(\gamma_{i+1}^{2}\right)=\operatorname{sgn}\left(y_{i+1}-y_{i}\right) .
$$

In the last case $\left(a_{2}, b_{2}\right)$, we get

$$
\operatorname{sgn}\left(C_{0}\left(\gamma_{i+2}^{3}-\gamma_{i+1}^{3}\right)\right)=\operatorname{sgn}\left(\gamma_{i+1}^{3}-\gamma_{i+2}^{3}\right)=-\operatorname{sgn}\left(\gamma_{i+2}^{3}\right)=\operatorname{sgn}\left(\gamma_{i+1}^{2}-\gamma_{i+2}^{2}\right)=\operatorname{sgn}\left(\gamma_{i+1}^{2}\right)=\operatorname{sgn}\left(y_{i+1}-y_{i}\right) .
$$


Case 5. Assume the stencils $S_{i}=\left\{C_{i-1}, C_{i}, C_{i+1}\right\}, S_{i+1}=\left\{C_{i+1}, C_{i+2}, C_{i+3}\right\}$, equivalent to the conditions

$$
\begin{gathered}
\begin{cases}\left|\gamma_{i}^{2}\right|<\left|\gamma_{i+1}^{2}\right|, & \left|\gamma_{i}^{3}\right| \geqslant\left|\gamma_{i+1}^{3}\right|, \quad(a) \\
\left|\gamma_{i}^{2}\right| \geqslant\left|\gamma_{i+1}^{2}\right|, & \left|\gamma_{i+1}^{3}\right|<\left|\gamma_{i+2}^{3}\right|, \quad(b)\end{cases} \\
\left|\gamma_{i+1}^{2}\right|>\left|\gamma_{i+2}^{2}\right|, \quad\left|\gamma_{i+2}^{3}\right|>\left|\gamma_{i+3}^{3}\right| .
\end{gathered}
$$

The jump is represented as

$$
j R_{i+1 / 2} \approx C_{0}\left(\gamma_{i+2}^{3}-\gamma_{i+1}^{3}\right)+C_{1}\left(\gamma_{i+3}^{3}-\gamma_{i+2}^{3}\right) .
$$

In the case of $(a)$ we precalculate

$$
\begin{gathered}
\operatorname{sgn}\left(-\gamma_{i+2}^{3}\right)=\operatorname{sgn}\left(\gamma_{i+1}^{2}-\gamma_{i+2}^{2}\right)=\operatorname{sgn}\left(\gamma_{i+1}^{2}\right), \\
\operatorname{sgn}\left(\gamma_{i+1}^{3}\right)=\operatorname{sgn}\left(\gamma_{i+1}^{2}-\gamma_{i}^{2}\right)=\operatorname{sgn}\left(\gamma_{i+1}^{2}\right) .
\end{gathered}
$$

With these we have

$$
\begin{aligned}
& \operatorname{sgn}\left(C_{0}\left(\gamma_{i+2}^{3}-\gamma_{i+1}^{3}\right)\right)=\operatorname{sgn}\left(\gamma_{i+1}^{3}-\gamma_{i+2}^{3}\right)=\operatorname{sgn}\left(\gamma_{i+1}^{2}\right)=\operatorname{sgn}\left(y_{i+1}-y_{i}\right), \\
& \operatorname{sgn}\left(C_{1}\left(\gamma_{i+3}^{3}-\gamma_{i+2}^{3}\right)\right)=\operatorname{sgn}\left(\gamma_{i+3}^{3}-\gamma_{i+2}^{3}\right)=-\operatorname{sgn}\left(\gamma_{i+2}^{3}\right)=\operatorname{sgn}\left(\gamma_{i+1}^{2}-\gamma_{i+2}^{2}\right)=\operatorname{sgn}\left(\gamma_{i+1}^{2}\right)=\operatorname{sgn}\left(y_{i+1}-y_{i}\right) .
\end{aligned}
$$

For $(b)$ we can use the same calculation as above for the second term since we were not using $(a)$. The sign of the first term is

$$
\operatorname{sgn}\left(C_{0}\left(\gamma_{i+2}^{3}-\gamma_{i+1}^{3}\right)\right)=\operatorname{sgn}\left(\gamma_{i+1}^{3}-\gamma_{i+2}^{3}\right)=\operatorname{sgn}\left(\gamma_{i+1}^{3}\right)=\operatorname{sgn}\left(\gamma_{i+1}^{2}-\gamma_{i+2}^{2}\right)=\operatorname{sgn}\left(\gamma_{i+1}^{2}\right)=\operatorname{sgn}\left(y_{i+1}-y_{i}\right) .
$$

Case 6. The last configuration is $S_{i}=\left\{C_{i}, C_{i+1}, C_{i+2}\right\}, S_{i+1}=\left\{C_{i+1}, C_{i+2}, C_{i+3}\right\}$, equivalent to the conditions

$$
\left|\gamma_{i}^{2}\right|>\left|\gamma_{i+1}^{2}\right|, \quad\left|\gamma_{i+1}^{3}\right|>\left|\gamma_{i+2}^{3}\right|, \quad\left|\gamma_{i+1}^{2}\right|>\left|\gamma_{i+2}^{2}\right|, \quad\left|\gamma_{i+2}^{3}\right|>\left|\gamma_{i+3}^{3}\right|
$$

with a reconstructed jump of the form

$$
j R_{i+1 / 2} \approx C_{0}\left(\gamma_{i+3}^{3}-\gamma_{i+2}^{3}\right) .
$$

We recover

$$
\operatorname{sgn}\left(C_{0}\left(\gamma_{i+3}^{3}-\gamma_{i+2}^{3}\right)\right)=\operatorname{sgn}\left(\gamma_{i+3}^{3}-\gamma_{i+2}^{3}\right)=-\operatorname{sgn}\left(\gamma_{i+2}^{3}\right)=\operatorname{sgn}\left(\gamma_{i+1}^{2}-\gamma_{i+2}^{2}\right)=\operatorname{sgn}\left(\gamma_{i+1}^{2}\right)=\operatorname{sgn}\left(y_{i+1}-y_{i}\right) .
$$

This completes the proof of the sign-property of the reconstruction method for grids as $\Delta x \rightarrow 0$ or the shape parameter $\varepsilon \rightarrow 0$.

\section{Appendix B. NEW Diffusion MATRIX}

The goal is to recover a new diffusion matrix that mimics the first order Rusanov-type diffusion operator. We combine results from Chandrashekar [5]

$$
\begin{aligned}
& \Delta v_{1}=\frac{\Delta \rho}{\hat{\rho}}+\left[\frac{1}{(\gamma-1) \hat{\beta}}-\overline{u^{2}}\right]-2 \bar{u} \bar{\beta} \Delta u \\
& \Delta v_{2}=2 \bar{\beta} \Delta u+2 \bar{u} \Delta \beta \\
& \Delta v_{3}=-2 \Delta \beta
\end{aligned}
$$


with the following

$$
\begin{aligned}
\Delta E & =\frac{\Delta p}{\gamma-1}+\frac{1}{2} \Delta\left(u^{2} \rho\right), \\
\frac{\Delta \rho}{2} & =\Delta(\beta p)=\bar{p} \Delta \beta+\bar{\beta} \Delta p, \\
\Delta\left(u^{2} \rho\right) & =2 \bar{\rho} \bar{u} \Delta u+\overline{u^{2}} \Delta \rho .
\end{aligned}
$$

This can be summarized as

$$
\left(\begin{array}{c}
\Delta \rho \\
\Delta m \\
\Delta E
\end{array}\right)=\tilde{D}\left(\begin{array}{l}
\Delta v_{1} \\
\Delta v_{2} \\
\Delta v_{3}
\end{array}\right)
$$

with

$$
\tilde{D}=\left(\begin{array}{ccc}
\hat{\rho} & \bar{u} \hat{\rho} & E_{1} \\
\bar{u} \hat{\rho} & \bar{u}^{2} \hat{\rho}+\frac{\bar{\rho}}{2 \bar{\beta}} & \bar{u} E_{1}+\frac{\bar{u}}{2 \bar{\rho}} \\
E_{2} & \bar{u} E_{2}+\frac{\bar{u} \bar{\rho}}{2 \bar{\beta}} & \tilde{R}
\end{array}\right),
$$

with $E_{1}=\hat{\rho} \bar{u}^{2}+\frac{\hat{\rho}}{2}\left[\frac{1}{(\gamma-1) \hat{\beta}}-\overline{u^{2}}\right], E_{2}=\frac{\hat{\rho}}{2}\left[\frac{1}{(\gamma-1) \bar{\beta}}+\overline{u^{2}}\right], \tilde{R}=\frac{E_{1} E_{2}}{\hat{\rho}}+\frac{\bar{u}^{2} \bar{\rho}}{2 \bar{\beta}}+\frac{\bar{p}}{2(\gamma-1) \bar{\beta}}$.

To use this in the entropy stable framework we need to symmetrize it. By assuming $\tilde{D}_{3,1}=E_{1}$ and $\tilde{D}_{3,2}=$ $\bar{u} E_{1}+\frac{\bar{u}}{2 \bar{\rho}}$ we get at least the exact jump for the density and mass flow.

We recover the matrix

$$
D=\alpha\left(\begin{array}{ccc}
\hat{\rho} & \bar{u} \hat{\rho} & E_{1} \\
\bar{u} \hat{\rho} & \bar{u}^{2} \hat{\rho}+\frac{\bar{\rho}}{2 \bar{\beta}} & \bar{u} E_{1}+\frac{\bar{u} \bar{\rho}}{2 \bar{\beta}} \\
E_{1} \bar{u} E_{1}+\frac{\bar{u}}{2 \overline{\bar{\beta}}} & R
\end{array}\right),
$$

with $R=\frac{E_{1}^{2}}{\hat{\rho}}+\frac{\bar{u}^{2} \bar{\rho}}{2 \bar{\beta}}+\frac{\bar{p}}{2(\gamma-1) \bar{\beta}}$, which mimics the Rusanov-type diffusion operator for the density and mass flow. By showing that the leading principal minors are positive we get the positive definiteness for $D$ in the case $\gamma>1$. However, we are not aware of an exact and stable decomposition $D=L B L^{T}$ for an invertable matrix $L$ and a diagonal one $B$. Thus, this needs to be done numerically in each step.

Note that Derigs et al. obtained a related result with a different value $R[6]$.

Acknowledgements. The authors are grateful to Dr. Deep Ray for helpful discussions and insights. This work was partially supported by SNSF.

\section{REFERENCES}

[1] T. Aboiyar, E.H. Georgoulis and A. Iske, High order weno finite volume schemes using polyharmonic spline reconstruction. In: Proceedings of the International Conference on Numerical Analysis and Approximation Theory NAAT2006. Dept. of Mathematics. University of Leicester, Cluj-Napoca (Romania) (2006).

[2] T. Aboiyar, E.H. Georgoulis and A. Iske, Adaptive ader methods using kernel-based polyharmonic spline weno reconstruction. SIAM J. Sci. Comput. 32 (2010) 3251-3277.

[3] C. Bigoni and J.S. Hesthaven, Adaptive weno methods based on radial basis function reconstruction. J. Sci. Comput. 72 (2017) 986-1020.

[4] J.P. Boyd, Error saturation in gaussian radial basis functions on a finite interval. J. Comput. Appl. Math. 234 (2010) $1435-1441$.

[5] P. Chandrashekar, Kinetic energy preserving and entropy stable finite volume schemes for compressible euler and navier-stokes equations. Commun. Comput. Phys. 14 (2013) 1252-1286.

[6] D. Derigs, A.R. Winters, G.J. Gassner and S. Walch, A novel averaging technique for discrete entropy-stable dissipation operators for ideal MHD. J. Comput. Phys. 330 (2017) 624-632.

[7] T.A. Driscoll and B. Fornberg, Interpolation in the limit of increasingly flat radial basis functions. Comput. Math. Appl. 43 (2002) 413-422.

[8] J. Duchon, Splines Minimizing Rotation-invariant Semi-norms in Sobolev Spaces. Springer, Berlin (1977) 85-100. 
[9] G.E. Fasshauer and J.G. Zhang, On choosing "optimal" shape parameters for RBF approximation. Numer. Algorithms 45 (2007) 345-368.

[10] U.S. Fjordholm, S. Mishra and E. Tadmor, Well-balanced and energy stable schemes for the shallow water equations with discontinuous topography. J. Comput. Phys. 230 (2011) 5587-5609.

[11] U.S. Fjordholm, S. Mishra and E. Tadmor, Arbitrarily high-order accurate entropy stable essentially nonoscillatory schemes for systems of conservation laws. SIAM J. Numer. Anal. 50 (2012) 544-573.

[12] U.S. Fjordholm, S. Mishra and E. Tadmor, Eno reconstruction and eno interpolation are stable. Found. Comput. Math. 13 (2013) 139-159.

[13] U.S. Fjordholm and D. Ray, A sign preserving weno reconstruction method. J. Sci. Comput. 68 (2016) $42-63$.

[14] B. Fornberg, E. Larsson and N. Flyer, Stable computations with gaussian radial basis functions in 2-D. Technical report, Department of Information Technology, Uppsala University (2009).

[15] B. Fornberg and C. Piret, A stable algorithm for flat radial basis functions on a sphere. SIAM J. Sci. Comput. 30 (2007) 60-80.

[16] B. Fornberg and G. Wright, Stable computation of multiquadric interpolants for all values of the shape parameter. Comput. Math. Appl. 48 (2004) 853-867.

[17] S. Gottlieb, D.I. Ketcheson and C.-W. Shu, High order strong stability preserving time discretizations. J. Sci. Comput. 38 (2009) 251-289.

[18] A. Harten, B. Engquist, S. Osher and S.R. Chakravarthy, Uniformly high order accurate essentially non-oscillatory schemes, III. J. Comput. Phys. 71 (1987) 231-303.

[19] A. Harten, J.M. Hyman, P.D. Lax and B. Keyfitz, On finitedifference approximations and entropy conditions for shocks. Commun. Pure Appl. Math. 29 (1976) 297-322.

[20] J.S. Hesthaven, Numerical Methods for Conservation Laws: From Analysis to Algorithms. Society for Industrial and Applied Mathematics, Philadelphia, PN (2018).

[21] S.N. Kružkov, First order quasilinear equations in several independent variables. Math. USSR-Sbornik 10 (1970) 217.

[22] E. Larsson and B. Fornberg, Theoretical and computational aspects of multivariate interpolation with increasingly flat radial basis functions. Comput. Math. Appl. 49 (2005) 103-130.

[23] A.-Y. le Roux, A numerical conception of entropy for quasi-linear equations. Math. Comput. 31 (1977) $848-872$.

[24] P.G. Lefloch, J.-M. Mercier and C. Rohde, Fully discrete, entropy conservative schemes of arbitraryorder. SIAM J. Numer. Anal. 40 (2002) 1968-1992.

[25] R.J. LeVeque, Numerical Methods for Conservation Laws. Springer Science \& Business Media, Berlin (1992).

[26] M.L. Merriam, An Entropy-based Approach to Nonlinear Stability. Stanford University, Stanford, CA, USA (1989).

[27] C.A. Micchelli, Interpolation of scattered data: distance matrices and conditionally positive definite functions. Constr. Approx. 2 (1986) 11-22.

[28] M.S. Mock, Systems of conservation laws of mixed type. J. Differ. Equ. 37 (1980) 70-88.

[29] G. Mühlbach, A recurrence formula for generalized divided differences and some applications. J. Approx. Theory 9 (1973) 165-172.

[30] G. Mühlbach, The general neville-aitken-algorithm and some applications. Numer. Math. 31 (1978) 97-110.

[31] F.J. Narcowich and J.D. Ward, Norm estimates for the inverses of a general class of scattered-data radial-function interpolation matrices. J. Approx. Theory 69 (1992) 84-109.

[32] D. Ray, P. Chandrashekar, U.S. Fjordholm and S. Mishra, Entropy stable scheme on two-dimensional unstructured grids for euler equations. Commun. Comput. Phys. 19 (2016) 1111-1140.

[33] S. Rippa, An algorithm for selecting a good value for the parameter c in radial basis function interpolation. Adv. Comput. Math. 11 (1999) 193-210.

[34] R. Schaback, Error estimates and condition numbers for radial basis function interpolation. Adv. Comput. Math. 3 (1995) 251-264.

[35] R. Schaback, Native hilbert spaces for radial basis functions I. New Deve. Approx. Theory 132 (1998) $255-282$.

[36] R. Schaback, Multivariate interpolation by polynomials and radial basis functions. Constr. Approx. 21 (2005) $293-317$.

[37] C.-W. Shu and S. Osher, Efficient implementation of essentially non-oscillatory shock-capturing schemes. J. Comput. Phys. 77 (1988) 439-471.

[38] E. Tadmor, The numerical viscosity of entropy stable schemes for systems of conservation laws. I. Math. Comput. 49 (1987) 91-103.

[39] B. Van Leer, Towards the ultimate conservative difference scheme. V. A second-order sequel to godunov's method. J. Comput. Phys. 32 (1979) 101-136.

[40] H. Wendland, Scattered Data Approximation. Cambridge University Press, Cambridge (2004).

[41] P. Woodward and P. Colella, The numerical simulation of two-dimensional fluid flow with strong shocks. J. Comput. Phys. 54 (1984) 115-173.

[42] G.B. Wright and B. Fornberg, Stable computations with flat radial basis functions using vector-valued rational approximations. J. Comput. Phys. 331 (2017) 137-156. 